TRABAJOS DE PREHISTORIA

54, n. $^{\circ} 1,1997$, pp. $35-56$

\title{
¿NOS PASAMOS \\ DE LA RAYA?: LA FRONTERA \\ HISPANO-PORTUGUESA \\ A TRAVÉS DE \\ LAS PUBLICACIONES \\ DE PREHISTORIA Y \\ PROTOHISTORIA
}

\author{
CROSSING THE LINE? \\ THE SPANISH-PORTUGUESE FRONTIER SEEN \\ THROUGH PUBLICATIONS \\ ON PRE- AND PROTOHISTORY
}

\author{
ÁNGELES GARCÍA MARÍN (*) \\ ÁNGEL L. RODRÍGUEZ ALCALDE (**) \\ M. ${ }^{a}$ JESÚS SAN MILLÁN BUJANDA (*) \\ GREGORIO DE VICENTE BOBADILLA $(* * *)$ \\ M. ${ }^{a}$ ISABEL MARTÍNEZ NAVARRETE $(* * * *)$
}

\section{RESUMEN}

Definimos las pautas de estudio de los arqueólogos españoles y portugueses y la comunicación entre ellos -y con colegas de otros países- a partir de una muestra de publicaciones especializadas ibéricas. Combinando el análisis de fuentes y citas, evaluamos cómo se investiga y sobre que territorio. Los resultados obtenidos facilitan indicadores bibliométricos sensibles a la especificidad de la Arqueología. No se advierten diferencias relevantes entre los arqueólogos ibéricos. El localismo determina la práctica ausencia de colaboración internacional y desanima la interregional. Favorece una escasa coautoría y el predominio de autores nacionales en las fuentes y del idioma nacional en la cita. La principal comunicación in-

(*) Dpto. de Documentación Científica y Análisis Bibliométrico en Ciencias Humanas. Área de Arqueología y Prehistoria. CINDOC. CSIC. Pinar, 25. 28006 Madrid. Correo electrónico Sanmillan@cindoc.csic.es

(*) Dpto. de Prehistoria. Facultad de Geografía e Historia. Universidad Complutense. 28040 Madrid.

$(* * *)$ Servicio de Informática. CINDOC. CSIC. Pinar, 25. 28006 Madrid.

(****) Dpto. de Prehistoria. Centro de Estudios Históricos. CSIC. Serrano, 13. 28001 Madrid. Correo electrónico cehmn1g@fresno.csic.es.

El artículo fue remitido en su versión final el 26-V-97. ternacional es la transfronteriza, seguida de la mantenida con la arqueología francesa y alemana de larga implantación en España y Portugal. La similaridad de la arqueología prerromana a uno y otro lado de «la raya» procede de su dependencia tradicional de la historia-cultural. Se denuncian los riesgos de manipulación nacionalista implícitos en una práctica científica que ajusta el ámbito de estudio y la cita a autores y publicaciones a los límites administrativos actuales.

\begin{abstract}
Using a sample of specialist publications from the Iberian Peninsula, we define the research patterns of Spanish and Portuguese archaeologists and the communication they maintain among themselves and with colleagues from other countries. Combining analyses of sources and citations, we evaluate how and where research is carried out. Our results help define bibliometric indicators responsive to the specific needs of Archaeology. We note the absence of important differences between Iberian archaeologists. They share a localism that causes a near absence of international collaboration and discourages cooperation between regions. Co-authored
\end{abstract}


works are rare, and in each country national authors are favored in the source and the national language in the citations. Most international communication occurs between Spain and Portugal, followed by links to French and German archaeologies, both long-established in the Peninsula. The similarity of pre-Roman archaeology in both sides of the "line» is the result of their shared dependency on traditional culture history. We note the risks of nationalist manipulation implicit in a scientific practice that tailors the problems studied and the publications and authors cited to modern administrative boundaries.

Palabras clave: Arqueología. Prehistoria. Producción científica. Bibliometría. Metodología. Sociografía de la ciencia. Nacionalismo. Fronteras. España. Portugal.

Key words: Archaeology. Prehistory. Scientific production. Bibliometry. Methodology. Sociography of science. Nationalism. Frontiers. Iberia. Spain. Portugal.

\section{INTRODUCCIÓN}

La década de 1980 supone una llamada de atención sobre la influencia tanto de la teoría y metodología como de la sociología de la ciencia -incluyendo política y nacionalismo- en una investigación arqueológica hasta entonces fundamentalmente preocupada por los datos.

Este artículo aborda esa temática definiendo algunas de las pautas de transferencia de información entre los arqueólogos ibéricos a partir de una muestra de publicaciones especializadas de ambos países. Se busca, en primer lugar, evaluar cómo se investiga (cuestiones formales, referencias bibliográficas, autoría, lengua y nacionalidad, etc.) y, en segundo lugar, sobre que territorio. En relación con el primer objetivo, se indaga en que medida la frontera nacional ha podido individualizar cada comunidad científica, así como su permeabilidad a la del país vecino y a las de otros países. En cuanto al segundo, se determina la importancia relativa de las distintas divisiones territoriales contemporáneas.

La información que se maneja es las publicaciones aludidas, así como las citas y la cartografía que contienen.

La bibliometría (a veces llamada cienciometría) se ha configurado como «aquélla disciplina que analiza estadísticamente una determinada literatura científica, su evolución histórica, sus campos y desarrollos temáticos, así como sus au- tores y usos en el ámbito científico propio o próximo» (Ferreiro, 1993: 18).

La cartografía «conecta la creación de mapas y la utilización de los mismos en un sólo proceso» comunicativo en el que intervienen la realidad, el autor del mapa, el mapa y su perceptor. El mapa es un «instrumento de reflexión para el autor» (Matanzo, 1982: 15 y 17) y el mejor indicador de cómo el arqueólogo piensa el espacio. Si la comunicación es efectiva, el observador tendrá la misma percepción de la realidad que el autor.

A su vez, al considerar en las pautas de publicación la lengua del documento citado y la nacionalidad de sus autores aportamos elementos al debate sobre la relevancia de las distintas tradiciones disciplinares e idiomas en la arqueología europea (Ruiz Zapatero, 1994).

El estudio sólo facilita algunos datos de carácter diacrónico. Por haberse primado mas otros aspectos en la selección de la muestra que la fecha de publicación de los documentos fuente, los datos cronológicos son desiguales.

\section{LA MUESTRA}

La búsqueda en bases de datos internacionales (Social Sciences Citation Index, 1989 a 1995 y Arts \& Humanities Citation Index, 1987 a 1995; Ulrich's, 1995; Francis. Bulletin Signaletique. Série Préhistorie et Protohistoire, 1990 a 1993) y la opinión de expertos designaron las primeras publicaciones (Lillios, 1995b: VI; Chapman, 1996: 33). No obstante, la selección estuvo abierta durante gran parte del proceso, interviniendo los sucesivos resultados del análisis bibliométrico en la muestra definitiva (Tab. 1).

La muestra de las publicaciones españolas toma como base un estudio previo sobre las mismas (Rodríguez et alii, 1996) y la proximidad a la frontera de sus lugares de edición. En Portugal, se tienen en cuenta los resultados de las consultas antes citadas y la vinculación entre las publicaciones y los centros regionales de investigación para dar una visión mas descentralizada. Se incluye Trabajos de Prehistoria (Madrid) por el elevado número de citas que recibe desde las revistas portuguesas a pesar de quedar fuera de la zona delimitada.

Los tipos de fuentes escogidas han sido «Revistas y Series monográficas», «Congresos» y «Monografías» por ser los mas representativos de

T. P., 54, n. ${ }^{\circ} 1,1997$ 


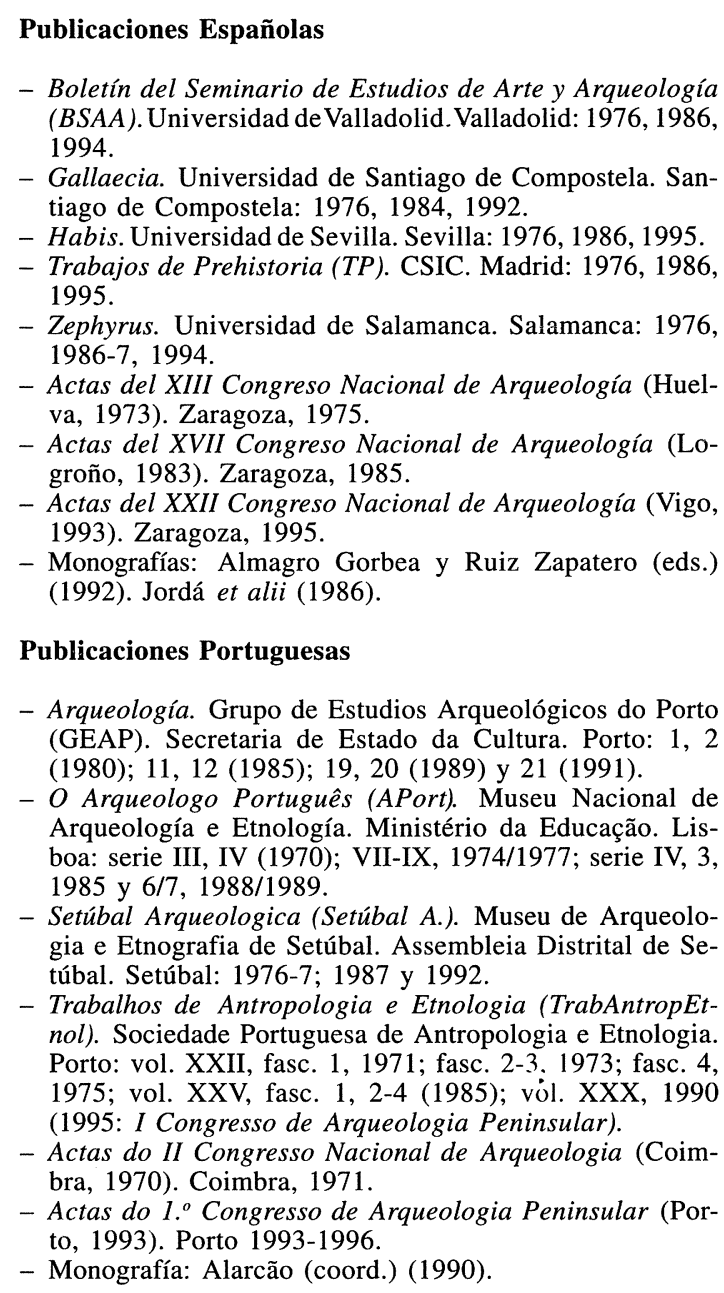
(BSAA). Universidad de Valladolid.Valladolid: 1976, 1986 1994.

- Gallaecia. Universidad de Santiago de Compostela. Santiago de Compostela: 1976, 1984, 1992.

- Habis. Universidad de Sevilla. Sevilla: 1976, 1986, 1995.

- Trabajos de Prehistoria (TP). CSIC. Madrid: 1976, 1986, 1995.

- Zephyrus. Universidad de Salamanca. Salamanca: 1976, 1986-7, 1994.

- Actas del XIII Congreso Nacional de Arqueología (Huelva, 1973). Zaragoza, 1975.

- Actas del XVII Congreso Nacional de Arqueología (Logroño, 1983). Zaragoza, 1985.

- Actas del XXII Congreso Nacional de Arqueología (Vigo, 1993). Zaragoza, 1995.

- Monografías: Almagro Gorbea y Ruiz Zapatero (eds.) (1992). Jordá et alii (1986).

Publicaciones Portuguesas

- Arqueología. Grupo de Estudios Arqueológicos do Porto (GEAP). Secretaria de Estado da Cultura. Porto: 1, 2 (1980); 11, 12 (1985); 19, 20 (1989) y 21 (1991).

- O Arqueologo Português (APort). Museu Nacional de Arqueología e Etnología. Ministério da Educação. Lisboa: serie III, IV (1970); VII-IX, 1974/1977; serie IV, 3, 1985 y $6 / 7,1988 / 1989$

- Setúbal Arqueologica (Setúbal A.). Museu de Arqueologia e Etnografia de Setúbal. Assembleia Distrital de Setúbal. Setúbal: 1976-7; 1987 y 1992.

- Trabalhos de Antropologia e Etnologia (TrabAntropEtnol). Sociedade Portuguesa de Antropologia e Etnologia. Porto: vol. XXII, fasc. 1, 1971; fasc. 2-3. 1973; fasc. 4 1975; vol. XXV, fasc. 1, 2-4 (1985); voll. XXX, 1990 (1995: I Congresso de Arqueologia Peninsular).

- Actas do II Congresso Nacional de Arqueologia (Coimbra, 1970). Coimbra, 1971.

- Actas do 1. ${ }^{\circ}$ Congresso de Arqueologia Peninsular (Porto, 1993). Porto 1993-1996.

- Monografía: Alarcão (coord.) (1990).

Tabla 1. Fuentes estudiadas.

las distintas pautas de comunicación científica. Además, en los dos últimos, se busca evaluar la influencia sobre dichas pautas de un ámbito definido por el territorio nacional o por el ibérico.

Se consideran únicamente los artículos, comunicaciones o capítulos relativos a Prehistoria y Protohistoria. Se quiere resaltar, mediante la discontinuidad de estos períodos históricos respecto al presente, los sesgos introducidos por la conceptualización actualista del territorio.

Se estudian las tres últimas décadas iniciadas con importantes cambios políticos en ambos países. En las revistas se seleccionaron los volúmenes desde el presente (1995), salvo que lo impidiera la falta de normalización en la periodicidad o la publicación de congresos (por ejemplo, Setú- bal A. 1975). En esos casos, se sustituyeron por los que permitieran una serie equivalente.

En los congresos españoles la fecha se cruzó con el lugar de celebración. Se han vaciado más actas españolas que portuguesas para tratar de paliar el escaso número de comunicaciones sobre la zona occidental ibérica en las primeras. La inclusión de las Actas del Congreso de Coimbra combina la opinión de expertos y el resultado del análisis de citas. Los volúmenes del Congreso de Oporto revisados eran los accesibles en las bibliotecas de Madrid en el momento de realización del estudio.

La selección de monografías responde también a la opinión de expertos y al impacto de las mismas. En el caso de la síntesis peninsular (Almagro Gorbea y Ruiz Zapatero, 1992) y de las Actas del Congreso de Oporto se han estudiado de forma independiente las colaboraciones de autores españoles y portugueses.

Para maximizar la recogida de datos (tiempo y normalización) uno de nosotros realizó una aplicación en dBase ${ }^{\circledR} \mathrm{V}$ consistente en tres tablas (citas, documentos fuente, títulos de revista) relacionadas (Rodríguez et alii, 1996: 41).

Se recogieron citas a siete tipos de documentos (Tab. 2) (Rodríguez et alii, 1996: 40), introduciendo en «Informes», los inéditos, los 'trabalhos' para las pruebas de capacitación pedagógica y científica y los 'ordenamentos' y en «Otros» las memorias de los mapas y los diccionarios.

Las «Revistas y Series monográficas» han recibido un tratamiento especial por su importancia en la comunicación científica. El uso de los ficheros de la biblioteca conjunta «Colección de Prehistoria» (CSIC) y del Museo Arqueológico Nacional, de repertorios bibliográficos y catálogos colectivos como Aleph (Catálogo colectivo automatizado de la red de bibliotecas del CSIC), ARIADNA (Biblioteca Nacional de España) y el del Instituto da Biblioteca Nacional e do Livro (http://www.cam.ac.uk/Hytelnet/pt0/pt001.html) ha permitido identificar en gran parte la temática y los lugares de publicación de este tipo de documento.

Los datos de localización, normalmente ausentes de las citas, se han obtenido para todas las «Revistas y Series monográficas» de España y Portugal publicadas dentro y fuera de la Península Ibérica más Madrider Mitteilungen (Mainz) y Mélanges de la Casa de Velazquez (París), editadas por instituciones vinculadas con la investiga- 


\begin{tabular}{|c|c|c|c|c|c|c|c|c|c|c|c|c|c|c|c|c|c|c|}
\hline Documentos Fuente & $A$ & B & C & D & $E$ & $F$ & $\mathbf{G}$ & $\mathrm{H}$ & 1 & $\mathrm{~J}$ & $\mathbf{K}$ & $\mathrm{L}$ & $M$ & $\mathrm{~N}$ & 0 & $\mathbf{P}$ & $Q$ & $\mathbf{R}$ \\
\hline Arqueologia & 4 & 82 & 1263 & 707 & 172 & 289 & 13 & 233 & 345 & 138 & 24 & 3 & 13 & 13 & 125 & 82 & 31 & 7,7 \\
\hline APort & 4 & 22 & 363 & 212 & 91 & 59 & 14 & 48 & 110 & 33 & 2 & 1 & & 4 & 171 & 19 & 6 & 19,95 \\
\hline Setúbal A. & 3 & 27 & 529 & 299 & 39 & 210 & 12 & 38 & 147 & 69 & & 1 & 3 & 8 & 39 & 35 & 13 & 28,5 \\
\hline TrabAntropEtnol & 5 & 30 & 305 & 165 & 62 & 85 & 4 & 14 & 111 & 19 & 5 & & 1 & 1 & 105 & 17 & 12 & 17,46 \\
\hline$B S A A$ & 3 & 9 & 358 & 214 & 152 & 27 & 9 & 26 & 103 & 29 & 5 & 2 & & 3 & 107 & 17 & 11 & 28,1 \\
\hline Gallaecia & 3 & 20 & 292 & 169 & 103 & 14 & 3 & 49 & 96 & 15 & 6 & 5 & & 1 & 32 & 12 & 4 & 19,6 \\
\hline Habis & 3 & 12 & 498 & 263 & 203 & 2 & 12 & 46 & 171 & 56 & 3 & 2 & & 2 & 20 & 6 & 2 & 17,8 \\
\hline$T P$ & 3 & 10 & 557 & 326 & 234 & 31 & 3 & 58 & 163 & 54 & 5 & 3 & 4 & 3 & 206 & 22 & 1 & 19 \\
\hline Zephyrus & 3 & 34 & 926 & 569 & 468 & 16 & 20 & 65 & 258 & 69 & 11 & 4 & 1 & 4 & 145 & 37 & 6 & 11,8 \\
\hline II CNA. Coimbra & 1 & 26 & 273 & 152 & 17 & 119 & 4 & 12 & 88 & 28 & & & 8 & 3 & & & & 8,8 \\
\hline $1^{\circ} \mathrm{CAP}$. Porto & 3 & 39 & 1084 & 508 & 157 & 243 & 6 & 102 & 312 & 142 & 16 & 8 & & 6 & & & & 18,2 \\
\hline XIII CNA. Huelva & 1 & 18 & 101 & 65 & 41 & 8 & 1 & 15 & 31 & 4 & & & & 1 & & & & 9,5 \\
\hline XVII CNA. Logroño & 1 & 8 & 149 & 87 & 65 & 10 & 1 & 11 & 42 & 18 & 1 & & & 1 & & & & 10 \\
\hline XX\| CNA. Vigo & 1 & 48 & 769 & 359 & 239 & 56 & 12 & 52 & 297 & 71 & 12 & 5 & 8 & 10 & & & & 6 \\
\hline Alarcão (coord.) (1990) & 1 & 8 & 562 & 280 & 48 & 185 & 12 & 35 & 188 & 69 & 9 & 1 & 6 & & & & & 39 \\
\hline Jordá et alii (1986) & 1 & 10 & 338 & 195 & 161 & 3 & 3 & 28 & 96 & 43 & 2 & & & & & & & 52,8 \\
\hline \begin{tabular}{|l|} 
Almagro Gorbea y Ruiz \\
Zapatero (eds.) (1992)
\end{tabular} & 1 & 11 & 920 & 518 & 396 & 69 & 14 & 39 & 267 & 111 & 15 & & 5 & 4 & & & & 15,9 \\
\hline TOTALES & 41 & 414 & 9287 & 5088 & 2648 & 1426 & 143 & 871 & 2825 & 968 & 116 & 35 & \begin{tabular}{|l|l|}
49 \\
\end{tabular} & 64 & 950 & 247 & 86 & \\
\hline
\end{tabular}

Tabla 2. Datos del muestreo por tipos de documentos. A. Números vaciados; B. Artículos vaciados; C. Citas generadas; D. Citas a «Revistas y Series monográficas»; E. Citas a «Revistas y Series monográficas» españolas; F. Citas a «Revistas y Series monográficas» portuguesas; G. Citas a Madrider Mitteilungen; H. Citas a otras «Revistas y Series monográficas» extranjeras; I. Citas a «Monografías»; J. Citas a «Congresos»; K. Citas a «Tesis doctorales»; L. Citas a «Memorias de licenciatura»; M. Citas a «Informes»; N. Citas a «Otros tipos de documentos»; O. Citas recibidas desde los otros documentos vaciados; P. Autocitas a la propia revista; $\mathbf{Q}$. Autocitas de autor que son autocitas de revista; R. Media de páginas por escrito.

ción en ese ámbito. Los resultados se han agrupado por provincias en España y Regiones en Portugal. El resto de las publicaciones extranjeras se han estudiado por países.

La fecha de la publicación es la de la última edición citada. Se han excluido las obras 'en prensa'. Los autores clásicos se citan por el comentarista de la edición. Los demás se identifican con nombre completo y dos apellidos, siempre que sea posible, indicándose su nacionalidad y la lengua en la que escriben. En los documentos fuente se toman hasta un máximo de cinco autores, mientras en las citas sólo el primero.

Esta doble base de datos es fundamental para establecèr las conexiones internas y externas de los investigadores de España y Portugal.

La normalización del fichero de autores se ha visto dificultada por la forma inversa de citar los apellidos en España y Portugal, no siempre comprendida cuando la referencia se hace desde el otro país. Además, el mismo autor aparece identificado de forma variable en el mismo documento (Gonçalves, A.H.B.) sin que los índices onomásticos, repertorios bibliográficos y catálogos aludidos resuelvan siempre el problema: Gonçalves, António A. Huet de B. (Oliveira, 1985: 52); Gonçalves, António Huet de Bacelar (Kunst, 1995: 356).

La casuística anterior insinúa algunos de los graves problemas que se plantean en la realización de la base de datos (Rodríguez et alii, 1996: 40). Respecto a la forma de citar de los investigadores, los obstáculos derivan de la aparición de citas incompletas, erróneas o contradictorias. Además se combinan formas diversas de llamada en el texto (año y autor, op.cit., ibidem, nota al pie) con localizaciones variadas en el mismo, introduciendo algunos errores insalvables en la cuantificación.

Estos factores, unidos a la irregularidad en periodicidad, estructura y aspectos formales de «Revistas y Series monográficas», dificultan la realización de repertorios y penalizan la inclusión de la producción científica de ambos países en las bases de datos internacionales (García Marín et alii, 1996: 1000-1008).

En cuanto a la información cartográfica asumimos que «la gran ventaja del mapa con respecto a 
los demás medios de comunicación es que puede ser visualizado instantáneamente» (Matanzo, 1982: 15). En ese sentido, nuestra clasificación no ha tenido en cuenta el pie, ni el título del escrito.

Por otra parte, cuando un autor recurre a una información cartográfica específica está asumiendo que el lector puede no ser especialista en el tema y desconocer el territorio salvo a escala nacional. Cabe esperar, entonces, que la identificación del mapa evite topónimos históricos (Bracara Augusta) e incluya otros de capitales (Oporto, Lisboa, Sevilla..), accidentes geográficos de primer orden (Duero, Tajo, Sierra de la Estrella, Sistema Central..) y líneas de costa o límites administrativos claramente identificables por la delineación o la rotulación.

En consecuencia, nuestra clasificación valora la posibilidad o no de identificación inmediata. Después destaca si la identificación se hace mediante elementos del paisaje o centros y límites administrativos. En ese caso se establece cómo se relaciona con los segundos la información arqueológica. La aparición de la Península Ibérica y de la frontera hispano-portuguesa ha sido objeto de especial atención. La cartografía de un escrito que representa distintas capas de información de la misma zona se considera unitariamente. La clasificación se hace en orden creciente (de 0 a 4 ) y acumulativo:

$0 \quad$ No se identifica

1 Se identifica la zona sin límites administrativos

1A con topónimos

1B con accidentes geográficos

2 Se identifica la zona con límites administrativos

$2 \mathrm{~A}$ nacionales

2A1 se jerarquizan los límites con la rotulación o la delineación

2A2 no se jerarquizan

2B límites autonómicos (España) y los equivalentes en Portugal

2C provinciales

2D municipales o inferiores

3 Aparece mas de un objeto de estudio (yacimientos, piezas, areas de distribución) y su distribución trasciende los límites administrativos.

$3 \mathrm{~A}$ nacionales

3B límites autonómicos (España) y los equivalentes en Portugal
3C provinciales

3D municipales o inferiores

4 Se incluye toda la Península Ibérica o mas zonas.

4A núcleo de la información

4B ventana complementaria

\section{ANÁLISIS}

Se han definido las pautas de publicación de los arqueólogos de España y Portugal a partir de:

A. Los documentos fuente: tipo de documento, páginas por tipo y por título de revista, lengua en la que están escritos, y su relación con la de las citas. Además, la autoría se conecta con la nacionalidad de los firmantes, la lengua del documento y la de las citas. Finalmente, se estudia una muestra de la cartografía.

B. Las citas: se estudian los aspectos ya mencionados y se cuantifican por tipo de documento y por décadas. En las referidas a «Revistas y Series monográficas» se evalúan temática y lugar de publicación.

Los resultados de estos análisis se combinan para evaluar aspectos formales de las publicaciones científicas, así como otros correspondientes a la autoría, la lengua y la nacionalidad y el ámbito territorial de las mismas.

\subsection{Aspectos formales}

Se intenta averiguar si existen pautas específicas entre los tipos de documentos y, en su caso, si se relacionan con su publicación en España o Portugal (Tab. 2). Por ello el comentario se centra en «Revistas y Series monográficas», «Congresos» $\mathrm{y}$ «Monografías», que cuentan con una muestra representativa.

Se considera la extensión de los escritos y las referencias bibliográficas para todos los documentos fuente (lectura horizontal de la tabla) y el volumen de citas generadas por tipo de documento (lectura vertical). Finalmente se indica la actualización de las citas y las autocitas de revista.

El número medio de páginas (Tab. 2:R) normalmente se emplea para caracterizar la extensión de los escritos de los documentos fuente. En nuestro caso permite comparar los tipos de documentos entre sí (revistas, monografías, congresos, etc.) pero no los documentos fuente incluidos en cada tipo. 


\begin{tabular}{|c|c|c|c|c|c|c|c|c|c|c|c|c|c|c|c|c|c|c|c|c|}
\hline & \multicolumn{4}{|c|}{ Arqueologia } & \multicolumn{4}{|c|}{ APort } & \multicolumn{4}{|c|}{ Setúbal A. } & \multicolumn{4}{|c|}{ TrabAntropEtnol } & \multicolumn{4}{|c|}{ TOTALES } \\
\hline Décadas & $A$ & $B$ & C & $\mathrm{D}$ & $A$ & B & C & $\mathrm{D}$ & $A$ & $\mathrm{~B}$ & C & $D$ & $\mathrm{~A}$ & $B$ & C & $D$ & $\bar{A}$ & $\bar{B}$ & $\bar{c}$ & D \\
\hline 1906-1915 & & 4 & 5 & 1 & & & 3 & & & & 4 & 1 & & 6 & 4 & & & 10 & 16 & 2 \\
\hline $1916-1925$ & & 1 & 10 & 2 & & 4 & 6 & 1 & & 2 & 3 & & & 2 & 3 & 2 & & 9 & 22 & 5 \\
\hline $1926-1935$ & 3 & 7 & 10 & 6 & & 5 & 5 & 1 & & 4 & 2 & 1 & 2 & 16 & 8 & & 5 & 32 & 25 & 10 \\
\hline $1936-1945$ & 2 & 11 & 15 & 4 & 2 & 6 & 9 & 1 & 2 & 5 & 14 & & 1 & 4 & 6 & & 7 & 26 & 44 & 5 \\
\hline $1946-1955$ & 1 & 23 & 20 & 15 & 2 & 7 & 12 & 2 & & 11 & 13 & & & 11 & 15 & & 3 & 52 & 60 & 17 \\
\hline $1956-1965$ & 5 & 28 & 49 & 14 & 6 & 8 & 28 & 4 & 9 & 17 & 48 & 2 & 4 & 21 & 24 & & 24 & 74 & 149 & 20 \\
\hline $1966-1975$ & 23 & 67 & 66 & 63 & 22 & 37 & 52 & 27 & 33 & 40 & 72 & 15 & 6 & 15 & 22 & 4 & $\overline{84}$ & 159 & 212 & 109 \\
\hline $1976-1985$ & 65 & 121 & 258 & 88 & 1 & 35 & 46 & 8 & 17 & 30 & 67 & 13 & 5 & 19 & 51 & 4 & 88 & 205 & 422 & 113 \\
\hline $1986-1995$ & 30 & 62 & 58 & 37 & & 4 & 1 & 1 & 5 & 21 & 34 & 5 & 1 & 5 & 13 & 3 & 36 & 92 & 106 & 46 \\
\hline
\end{tabular}

\begin{tabular}{|c|c|c|c|c|c|c|c|c|c|c|c|c|c|c|c|c|c|c|c|c|c|c|c|c|}
\hline & \multicolumn{4}{|c|}{ BSAA } & \multicolumn{4}{|c|}{ Gallaecia } & \multicolumn{4}{|c|}{ Habis } & \multicolumn{4}{|c|}{$T P$} & \multicolumn{4}{|c|}{ Zephyrus } & \multicolumn{4}{|c|}{ TOTALES } \\
\hline Décadas & $A$ & $B$ & $\mathrm{C}$ & D & $A$ & $\mathrm{~B}$ & $\mathrm{C}$ & D & $A$ & $\mathrm{~B}$ & $\mathrm{C}$ & D & $A$ & $\mathrm{~B}$ & $\mathrm{C}$ & D & $\mathrm{A}$ & $\mathrm{B}$ & $\mathrm{C}$ & $D$ & A & $\mathrm{B}$ & $\mathrm{C}$ & D \\
\hline $1906-1915$ & & & 3 & 1 & & & 1 & & & 7 & 2 & 6 & & & 4 & & & 4 & 2 & 1 & & 11 & 12 & 8 \\
\hline $1916-1925$ & & 2 & 5 & & & & 2 & 1 & 1 & 6 & 14 & 2 & 1 & 2 & 24 & 1 & & 9 & 4 & 2 & 2 & 19 & 49 & 6 \\
\hline $1926-1935$ & & 4 & 7 & 1 & & 4 & 10 & 1 & & 8 & 12 & 4 & & 5 & 17 & 1 & 1 & 18 & 30 & 2 & 1 & 39 & 76 & 9 \\
\hline $1936-1945$ & & 1 & 6 & 1 & & 5 & 3 & & & 18 & 11 & & & 2 & 9 & 2 & & 12 & 16 & 1 & 0 & 38 & 45 & 4 \\
\hline 1946-1955 & 2 & 6 & 13 & 2 & 1 & 8 & 7 & & 6 & 10 & 13 & 2 & 6 & 5 & 11 & 1 & 4 & 24 & 34 & 3 & 19 & 53 & 78 & 8 \\
\hline $1956-1965$ & 5 & 14 & 30 & 2 & & 28 & 10 & & 10 & 21 & 37 & 7 & 4 & 12 & 29 & 10 & 12 & 47 & 57 & 8 & 31 & 122 & 163 & 27 \\
\hline $1966-1975$ & 9 & 21 & 49 & 9 & 3 & 23 & 31 & 13 & 33 & 38 & 60 & 18 & 18 & 25 & 39 & 17 & 19 & 64 & 104 & 33 & 82 & 171 & 283 & 90 \\
\hline 1976-1985 & 6 & 18 & 46 & 5 & 7 & 20 & 29 & 23 & 6 & 28 & 62 & 2 & 6 & 31 & 36 & 11 & 29 & 65 & 236 & 11 & 54 & 162 & 409 & 52 \\
\hline 1986-1995 & 7 & 34 & 32 & 4 & 4 & 8 & 26 & 11 & & 7 & 7 & 1 & 19 & 72 & 93 & 15 & 4 & 11 & 30 & 1 & 34 & 132 & 188 & 32 \\
\hline
\end{tabular}

Tabla 3. Evolución de las citas a cada tipo de documento por décadas: (A) «Congresos»; (B) «Monografías»; (C) «Revistas y Series Monográficas» ibéricas; (D) «Revistas y Series monográficas» extranjeras. Las diferencias respecto a los totales de la Tabla 2: F, G, H se deben a citas anteriores a 1906.

Como era previsible, las «Monografías» y los «Congresos» marcan los límites entre los que se sitúan las «Revistas y Series monográficas». La extensión de los artículos en las mismas depende del número de los publicados (Tab. 2:B) y varía notablemente de unas a otras. Ello debe ponerse en relación con la escasa normalización de las revistas a este respecto: los artículos de Setúbal Arqueologica y Arqueologia oscilan entre las 2 páginas y las 137 y 33 respectivamente, los de Zephyrus entre 2 y 28 páginas y los de Gallaecia entre 8 y 89. La edición en España o Portugal no resulta muy significativa y apenas insinúa una tendencia a reducir la extensión de los artículos para aumentar su número en el segundo. El ejemplo más claro es Arqueologia más parecida a ese respecto a las actas de congresos que a las otras revistas.

Cada tipo de documento tiene una media específica de citas por artículo (Tab. 2:B,C) con un máximo en las monografías y un mínimo en los congresos. Las únicas diferencias nacionales se advierten en las revistas, duplicando las españolas (31) a las portuguesas $(15,3)$ en número de referencias. Como, por otra parte, todas las revistas lusas se acercan a la media portuguesa, consideramos que este puede ser un criterio indicativo de una cierta tradición científica. Las revistas espa- ñolas son muy distintas entre sí teniendo Gallaecia valores análogos a las portuguesas.

La homogeneidad en la media de citas por artículo en las revistas portuguesas y las diferencias entre las españolas se observa también en la proporción de autocitas de revista respecto del total de citas a «Revistas y Series monográficas» (Tab. 2:P,D). Esas autocitas representan aproximadamente el doble en las portuguesas (11\%) que en las españolas (6\%).

El estudio de la evolución de las citas a cada tipo de documento (Tab. 3) debe tener en cuenta que, por las características de la muestra (Tab. 1), la última década está, generalmente, infrarrepresentada y, cuando el volumen más reciente es de los años 80, incluso la anterior.

Los tipos de documentos más citados son «Revistas y Series monográficas» seguido de «Monografías» y de «Congresos». Por ello, sólo nos referiremos a los dos primeros.

Un rasgo común a las publicaciones ibéricas, que comparten con revistas españolas (Rodríguez et alii, 1996: 55), es la actualización de la cita: en la década 1976-85 se concentra la tercera parte de las referencias, en tanto que toda la primera mitad del siglo apenas suma esta cantidad.

Existe una gran variedad entre las distintas revistas españolas y portuguesas en cuanto al ma- 
nejo de «Revistas y Series monográficas» no ibéricas (Tab. 3:D) de forma que no se puede utilizar este rasgo para su distinción. Sí se advierte un uso mayor de la cita a éstas desde las portuguesas que desde las españolas: $31 \%$ y $18 \%$, respectivamente, del total de citas a ese tipo de documento. Diacrónicamente, la cita sigue las pautas indicadas para las publicaciones ibéricas.

\subsection{Contenido de las «Revistas y Series monográficas»}

La temática se ha determinado según varios criterios: las palabras más significativas de los títulos de las publicaciones citadas, la consulta de los catálogos mencionados donde figuran la institución editora y la Clasificación Decimal Universal.

No se advierten diferencias significativas entre ambos países.

El primer grupo de materias corresponde al núcleo de la disciplina y recibe el mayor porcentaje de citas en ambos países (Tab. 4:A). Le sigue en importancia el cuarto. Incluye publicaciones generales de las universidades, de los museos y de instituciones locales. Está conectado con dicho núcleo, ya que esas publicaciones suelen incluir artículos de Prehistoria y Arqueología (Revista de Guimarães, Cuadernos de Estudios Gallegos). Los otros dos grupos identificados están representados por un número similar de títulos. En el de las Ciencias Naturales destaca la Geología con 15 títulos en España y 22 en Portugal que suponen prácticamente la mitad de este grupo. Las revistas de Ciencias sin especificar y de Historia Natural ocupan el segundo lugar. A su vez, en el de Humanidades predominan los títulos de Historia.

Interpretamos estos resultados como indicadores de un uso especializado de la bibliografía en el mismo sentido que hemos comentado en relación con las misceláneas.

Por último, relacionamos las publicaciones sin identificar con citas de principios de siglo o incorrectas. En cualquier caso, ninguna supera las tres citas.

Las revistas con diez o más citas son realmente las que definen las pautas de consulta bibliográfica (Anexo y Tab. 4:B). Los 274 títulos diferentes citados desde las fuentes españolas y los 284 desde las portuguesas cuantifican la dispersión de las publicaciones en Prehistoria y Ar-

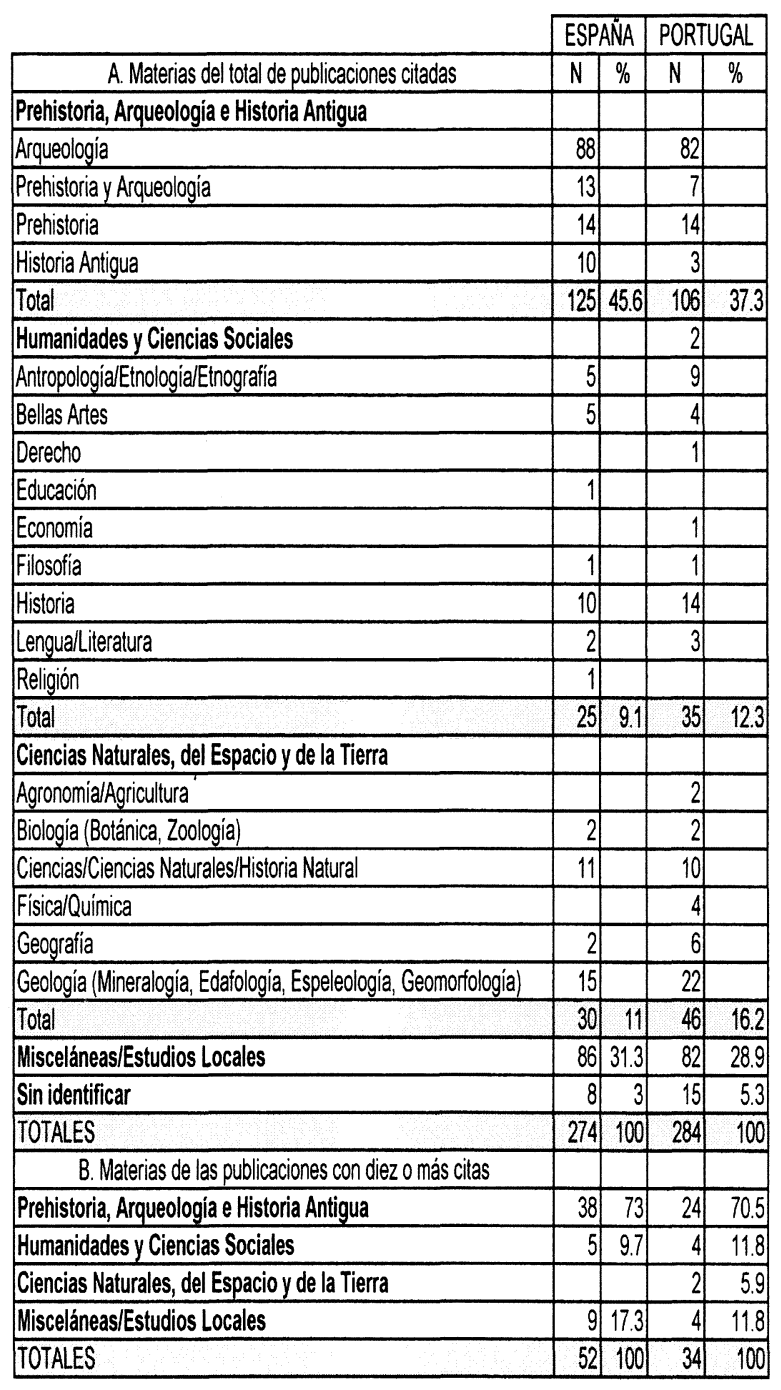

Tabla 4. Contenido de las «Revistas y Series monográficas» citadas.

queología. Para valorar su significación hay que tener en cuenta que 113 y 99 de dichos títulos sólo se citaron una vez y los 52 y 34 respectivamente que aparecen diez o más veces acumulan el $75 \%$ y el $67 \%$ del total de referencias.

La temática, tanto en España como en Portugal, mantiene la misma importancia relativa de todos los grupos que en el conjunto de las citas. En cambio, las publicaciones de Arqueología se duplican en detrimento de las misceláneas $\mathrm{y}$, mientras en España están ausentes las de Ciencias Naturales, en Portugal dos de Geología ocupan los puestos tercero y vigésimo de la clasificación.

Este núcleo de publicaciones caracteriza lo fun- 


\begin{tabular}{|l|c|c|c|c|c|c|c|c|c|c|c|c|c|c|}
\hline Documentos Fuente & $\mathbf{1}$ & $\mathbf{2}$ & $\mathbf{3}$ & $\mathbf{4}$ & $\mathbf{5}$ & $\mathbf{6}$ & $\mathbf{7}$ & $\mathbf{8}$ & $\mathbf{9}$ & $\mathbf{1 0}$ & $\mathbf{1 1}$ & $\mathbf{1 2}$ & $\mathbf{1 3}$ & $\mathbf{1 4}$ \\
\hline Arqueologia & 79 & 110 & 65 & 582 & 372 & 219 & 114 & 41 & 34 & 2 & 1 & 23 & 4 & \\
\hline APort & 33 & 48 & 22 & 199 & 130 & 51 & 29 & 8 & 6 & 1 & & 6 & & 1 \\
\hline Setúbal A. & 31 & 50 & 25 & 199 & 123 & 84 & 53 & 14 & 12 & & 1 & 3 & & 1 \\
\hline TrabAntropEtnol & 26 & 35 & 22 & 163 & 109 & 44 & 30 & 5 & 8 & & & & 1 & \\
\hline BSAA & 8 & 13 & 4 & 190 & 123 & 47 & 25 & 8 & 9 & 1 & 1 & 3 & & \\
\hline Gallaecia & 27 & 39 & 21 & 179 & 128 & 24 & 9 & 5 & 3 & 1 & 3 & 3 & & \\
\hline Habis & 12 & 12 & 12 & 216 & 147 & 13 & 8 & 3 & & 1 & 1 & & & \\
\hline TP & 13 & 13 & 13 & 268 & 170 & 57 & 24 & 14 & 14 & & 1 & 4 & & \\
\hline Zephyrus & 42 & 52 & 34 & 333 & 210 & 70 & 52 & 5 & 6 & 2 & 1 & 3 & & 1 \\
\hline II CNA. Coimbra & 27 & 38 & 21 & 123 & 75 & 59 & 36 & 10 & 11 & & & 1 & & 1 \\
\hline $1^{\circ}$ CAP. Porto & 70 & 73 & 67 & 530 & 338 & 138 & 51 & 40 & 22 & 6 & 6 & 11 & 2 & \\
\hline XIII CNA. Huelva & 25 & 25 & 25 & 67 & 51 & 9 & 5 & 1 & 3 & & & & & \\
\hline XVII CNA. Logroño & 7 & 8 & 7 & 87 & 61 & 20 & 9 & 5 & 4 & 1 & & 1 & & \\
\hline XXII CNA. Vigo & 52 & 63 & 44 & 358 & 240 & 86 & 44 & 17 & 15 & 1 & 2 & 1 & 4 & 2 \\
\hline Alarcão (coord.) (1990) & 4 & 8 & 2 & 209 & 110 & 18 & 9 & 1 & 7 & & & & 1 & \\
\hline Jordá et alii (1986) & 4 & 10 & 2 & 164 & 102 & 98 & 10 & 4 & 83 & & & 1 & & \\
\hline Almagro Gorbea y Ruiz & 20 & 20 & 20 & 336 & 189 & 89 & 59 & 17 & 5 & 3 & & 1 & 4 & \\
\hline Zapatero (eds.)(1992) & & & & & & & & & & & & & \\
\hline TOTALES & 480 & 617 & 406 & & & 1126 & 567 & 198 & 242 & 19 & 17 & 61 & 16 & 6 \\
\hline
\end{tabular}

Tabla 5. Datos del muestreo relativos a los autores 1. Autores por documento fuente; 2. Firmas por documento fuente; 3. Autores con un solo escrito; 4 . Autores citados; 5 . Autores citados una sola vez; $\mathbf{6}$. Total de autocitas de autor emitidas; 7. Autocitas de autor emitidas a «Revistas y Series monográficas» ibéricas; 8. Autocitas de autor emitidas a «Congresos»; 9. Autocitas de autor emitidas a «Monografías»; 10. Autocitas de autor emitidas a «Tesis doctorales»; 11. Autocitas de autor emitidas a «Memorias de licenciatura»; 12. Autocitas de autor emitidas a «Revistas y Series monográficas» extranjeras; 13. Autocitas de autor emitidas a «Informes»; 14. Autocitas de autor emitidas a «Otros tipos de documentos».

damental de la producción científica en estas disciplinas.Ahora bien, una historia de la ciencia que se restringiera al mismo podría dejar de lado parte de lo que esa producción debe a la especialización. En nuestro estudio hemos advertido la importancia del territorio, bien sea de la propia provincia, $\mathrm{Re}-$ gión u otro, y de la temática, fundamentalmente cronológica (Rodríguezet alii, 1996: 56).

La presencia de publicaciones españolas en la clasificación portuguesa es muy llamativa no sólo por el número de títulos (15) y de citas que reciben, sino por la cifra decepcionante de títulos portugueses en la lista española (5). Por lo menos, éstos tienen un importante número de citas...

\subsection{Autoría}

El estudio agrupa los documentos fuente por su nacionalidad. En los portugueses hay 211 au- tores distintos, que publican 234 escritos y en los españoles hay 177 autores que publican 182 (índices de coautoría 0,95 y 0,98 respectivamente) (1) (Tab. 5).

La presencia de cada autor en la investigación se fija a través del número de escritos que publica, el de citas que recibe y cuántas de ellas son autocitas. La referencia a la nacionalidad del autor permite estudiar la colaboración internacional.

Como, por un lado, puede haber varios firmantes de un escrito y, por otro, la misma persona puede figurar en varios de ellos, la producción científica de los autores se ha calculado individualizando hasta cinco cofirmantes y a partir del número total de firmas (en Portugal 363 y en España 255) y no del de autores distin-

(1) Índice de coautoría: relación número de firmas/número de trabajos ( $\left.\mathrm{F}^{*} \mathrm{~N} / \mathrm{N}\right)$ (Rodríguez Alcalde et alii, 1966: 45).

T. P., 54, n. $^{\circ} 1,1997$ 
tos (2). A su vez, en términos generales, la baja coautoría permite igualar el número de firmas con el de escritos.

Para que el volumen de datos fuera manejable, nos hemos centrado en los autores que han publicado tres o más escritos. Los autores con el mismo número se relacionan alfabéticamente.

Como puede verse en la tabla 6:D hay 16 autores en los documentos fuente españoles y 22 en los portugueses. M. F. dos Santos aparece en ambas series y es el único extranjero en la española. Hay que esperar hasta la posición 34 para encontrar el siguiente (Jose Manuel Fernandes Rolao con 2 artículos). Por el contrario, en la portuguesa hay 5 autores de tres nacionalidades.

La conexión entre la productividad de estos autores y su impacto se evalúa estudiando aquéllos que suman una tercera parte del total de citas emitidas (Tab. 6:B). Los que reciben el mismo número se relacionan alfabéticamente. Hay que tener en cuenta que en los documentos fuente portugueses se citan 1345 autores distintos de los cuales $804(60 \%)$ lo hacen una sola vez y en los españoles 1264 de los cuales $737(58,3 \%)$ también una sola vez (3).

Desde los documentos fuente portugueses 45 autores concentran ese tercio de citas y 41 desde los españoles. Los únicos autores no ibéricos que aparecen en ambas series son $\mathrm{Ph}$. Kalb, G. Leisner, H. Schubart y E. Anati. Los españoles son G. Delibes de Castro, M. Almagro Gorbea, M. Almagro Basch, F. Criado Boado, J.M. Vázquez Varela, P. Bosch Gimpera y F. López Cuevillas. La presencia internacional en la serie portuguesa es mayor que en la española (15 y 6 respectivamente). Por otra parte, desde los documentos portugueses se citan 7 autores españoles, pero desde los españoles no se cita ninguno portugués. En resumen los autores extranjeros citados desde los documentos portugueses son un tercio del total, mientras que desde los españoles sólo una sexta parte.

La especificidad de los ranking de autores desde cada serie de documentos fuente pone de manifiesto, en primer lugar, una frontera en lo que se refiere a las pautas de cita. En segundo lugar expresa con claridad la relatividad de cual-

(2) Por ejemplo F. Jordá, M. Pellier, P. Acosta y M. Almagro-Gorbea (1986) y M. Almagro Gorbea y G. Ruiz Zapatero (eds.) (1992) son cinco autores distintos pero seis firmas.

(3) Hay que tener en cuenta que un autor puede ser citado desde varios documentos. quier evaluación del impacto de la producción científica de un arqueólogo a partir únicamente del rango de citas que recibe. A este respecto el documento fuente y los límites cronológicos de la muestra son determinantes.

Finalmente, como rasgo común a las series ibéricas y entrando ya en la posición concreta de cada autor en las mismas destacamos que, entre los diez primeros autores mas citados, figuran algunos de los fundadores de la arqueología peninsular. Estos se identifican en la tabla 6, bien por su ausencia de los documentos fuente, bien por su fallecimiento con anterioridad a 1975 -quienes no tienen simultáneamente valores en las columnas D y C-, o bien porque publicaron menos de tres escritos, valor límite de la muestra.

La evaluación del impacto de la producción de otros autores queda penalizada por haber publicado recientemente. Otros la ven favorecida por su vinculación a la edición de los documentos fuente.

Todas esas circunstancias hacen que la comparación de los ranking de autores de las diferentes «Revistas y Series monográficas» de resultados notablemente divergentes.

Una última precisión matiza la presencia extranjera en los documentos fuente y en las citas. Los autores extranjeros que aparecen, salvo E. Anati, pueden considerarse nacionales a los efectos de la investigación (J. Roche, Ph. Kalb, H. Schubart..). Al menos parte de las citas desde los documentos portugueses a autores españoles y a otros extranjeros pueden deberse a su presencia como autores en las publicaciones portuguesas.

Un aspecto significativo de la autoría es el trabajo en equipo. Nos vamos a centrar en la colaboración internacional a partir de los documentos fuente. Esta tarea se ve seriamente obstaculizada por la escasa coautoría en la muestra. Es difícil decidir si su completa ausencia de ciertas publicaciones españolas (Habis; XVII CNA; Jordá et alii, 1986) está determinada o no por nuestros criterios de selección. En segundo lugar, donde se constata corresponde mayoritariamente a autores de la misma nacionalidad, en nuestro caso portugueses o españoles. Finalmente tanto esos equipos como los internacionales, normalmente están integrados por las mismas personas.

Sólo en 134 (32,3\%) del total de 414 documentos fuente hay coautoría. Centrándonos en los primeros, $44(32,8 \%)$ corresponden a publi- 


\begin{tabular}{|c|c|c|c|c|}
\hline Documentos fuente portugueses & A & $\mathbf{B}$ & C & D \\
\hline Jorge, Vítor Oliveira & PT & 112 & 21 & 14 \\
\hline Silva, Carlos Tavares da & $\mathrm{PT}$ & 90 & 34 & 13 \\
\hline Leisner, Georg & $\mathrm{AL}$ & 68 & & \\
\hline Ferreira, Octavio da Veiga & $\mathrm{PT}$ & 56 & 21 & 14 \\
\hline Paço, Afonso do & PT & 56 & 3 & \\
\hline Roche, Jean & $\mathrm{F}$ & 52 & 28 & 4 \\
\hline Jorge, Susana Oliveira & PT & 48 & 17 & 9 \\
\hline Leisner, Vera & $A L$ & 47 & 2 & \\
\hline Serrão, Eduardo da Cunha & $\mathrm{PT}$ & 47 & 13 & 4 \\
\hline Vasconcelos, Jose Leite de & PT & 44 & & \\
\hline Zbyszewski, George & $\mathrm{F}$ & 42 & 6 & 8 \\
\hline Cabral, João M. Peixoto & PT & 41 & 35 & \\
\hline Arnaud, Jose Morais & $\mathrm{PT}$ & 39 & 2 & \\
\hline Soares, Joaquina & $\mathrm{PT}$ & 38 & 17 & 11 \\
\hline Santos, Manuel Farinha dos & $\mathrm{PT}$ & 37 & 7 & 3 \\
\hline Schubart, Hermanfrid & $\mathrm{AL}$ & 37 & 3 & 3 \\
\hline Gonçalves, Victor dos Santos & $\mathrm{PT}$ & 34 & 6 & 3 \\
\hline Beirão, Caetano de Melo & $\mathrm{PT}$ & 32 & 3 & \\
\hline Kalb, Philine & $\mathrm{AL}$ & 32 & 6 & 3 \\
\hline Zilhão, João & $\mathrm{PT}$ & 32 & 2 & \\
\hline Breuil, Henri & $\mathrm{F}$ & 30 & & \\
\hline Junior, Joaquin R. dos Santos & PT & 30 & 16 & 5 \\
\hline Delibes de Castro, Germán & $E$ & 28 & 5 & \\
\hline Sangmeister, Eduard & $\mathrm{AL}$ & 26 & 17 & \\
\hline Almagro Gorbea, Martin & $E$ & 25 & & \\
\hline Anati, Emmanuel & 1 & 25 & & \\
\hline Soares, Antonio Manuel Monge & PT & 24 & 12 & 5 \\
\hline Viana, Abel & $\mathrm{PT}$ & 23 & 4 & \\
\hline Silva, Armando Coelho Ferreira da & $\mathrm{PT}$ & 22 & 7 & \\
\hline Almagro Basch, Martin & $E$ & 21 & & \\
\hline Criado Boado, Felipe & $E$ & 21 & 8 & \\
\hline Baptista, Antonio Martinho & $\mathrm{PT}$ & 20 & 5 & \\
\hline Gomes, Mario Varela & $\mathrm{PT}$ & 20 & 3 & 5 \\
\hline Nunes, João de Castro & $\mathrm{PT}$ & 20 & & \\
\hline Raposo, Luis & $\mathrm{PT}$ & 20 & & \\
\hline Rocha, Antonio dos Santos & PT & 20 & & \\
\hline Alarcão, Jorge de & $\mathrm{PT}$ & 19 & & \\
\hline Cardoso, João Luís & $\mathrm{PT}$ & 19 & 8 & 6 \\
\hline Vázquez Varela, José Manuel & $E$ & 19 & 5 & \\
\hline Oosterbeek, Luiz Miguel & PT & 18 & 12 & \\
\hline Almeida, Carlos Alberto Ferreira de & $\mathrm{PT}$ & 17 & 1 & \\
\hline Bosch Gimpera, Pere & $E$ & 17 & & \\
\hline López Cuevillas, Florentino & $E$ & 17 & & \\
\hline Senna-Martinez, Joao Carlos de & PT & 17 & & \\
\hline Silva, Fernando Augusto Pereira da & $\mathrm{PT}$ & 17 & 12 & \\
\hline \multicolumn{5}{|c|}{ Autores no citados } \\
\hline Leitão, Manuel & $\mathrm{PT}$ & & & 4 \\
\hline Monteiro, Jorge Pinho & $\mathrm{PT}$ & & & 4 \\
\hline North, C.T. & $\mathrm{RU}$ & & & 4 \\
\hline Sanches, Maria de Jesus & $\mathrm{PT}$ & & & 4 \\
\hline Guerra, Antonio Vitor & $\mathrm{PT}$ & & & 3 \\
\hline Moura, Maria Helena & $\mathrm{PT}$ & & & 3 \\
\hline Straus, Lawrence Guy & OT & & & 3 \\
\hline
\end{tabular}

\begin{tabular}{|c|c|c|c|c|}
\hline Documentos fuente españoles & A & B & $\mathbf{C}$ & D \\
\hline Maluquer de Motes, Joan & $E$ & 106 & & \\
\hline Almagro Gorbea, Martín & $E$ & 90 & 31 & 7 \\
\hline Almagro Basch, Martin & $E$ & 70 & & \\
\hline Martín Valls, Ricardo & $E$ & 67 & 35 & 5 \\
\hline Delibes de Castro, Germán & $E$ & 56 & 36 & 6 \\
\hline López Cuevillas, Florentino & $E$ & 52 & & \\
\hline Vázquez Varela, José Manuel & $E$ & 49 & 4 & 7 \\
\hline Bosch Gimpera, Pere & $E$ & 47 & & \\
\hline Cabré Aguiló, Juan & $E$ & 47 & & \\
\hline Schubart, Hermanfrid & $\mathrm{AL}$ & 47 & 1 & \\
\hline Blázquez Martínez, José María & $E$ & 46 & & \\
\hline Peña Santos, Antonio de la & $E$ & 46 & 23 & 5 \\
\hline Ruiz-Gálvez Priego, Marisa & $E$ & 45 & 2 & \\
\hline Palol Salellas, Pere de & $E$ & 44 & & \\
\hline Garcia Bellido, Antonio & $E$ & 43 & & \\
\hline Aubet Semmler, $\mathrm{M}^{2}$ Eugenia & $E$ & 41 & 4 & \\
\hline Criado Boado, Felipe & $E$ & 40 & & \\
\hline Pellicer Catalán, Manuel & $E$ & 40 & 11 & 4 \\
\hline Blanco Freijeiro, Antonio & $E$ & 39 & & \\
\hline Tovar Llorente, Antonio & $\bar{E}$ & 39 & & \\
\hline Wattenberg Sanpere, Federico & $E$ & 36 & & \\
\hline Beltrán Martínez, Antonio & $E$ & 33 & 17 & \\
\hline Cuadrado Diaz, Emeterio & $E$ & 32 & & \\
\hline Schulten, Adolf & $\mathrm{AL}$ & 32 & & \\
\hline Arribas Palau, Antonio & $E$ & 31 & & \\
\hline Perez de Barradas, José & $E$ & 31 & & \\
\hline Hidalgo Cuñarro, José Manuel & $E$ & 30 & 8 & 3 \\
\hline Leisner, Georg & $\overline{A L}$ & 30 & & \\
\hline Anati, Emmanuel & 1 & 29 & & \\
\hline Esparza Arroyo, Angel & $E$ & 28 & 2 & \\
\hline Rornero Carnicero, Fernando & $E$ & 28 & 7 & \\
\hline Carriazo, Juan de Mata & $E$ & 27 & & \\
\hline Fábregas Valcarce, Ramón & $E$ & 27 & 4 & 4 \\
\hline Eiroa Garcia, Jorge Juan & $E$ & 25 & & \\
\hline Morán Bardón, César & $E$ & 25 & & \\
\hline Schüle, Wilhem & $\mathrm{AL}$ & 25 & & \\
\hline Kalb, Philine & $\mathrm{AL}$ & 24 & 2 & \\
\hline Ruiz Mata, Diego & $E$ & 24 & 2 & \\
\hline Monteagudo, Luis & $E$ & 23 & & \\
\hline Albertos Firmat, $\mathrm{M}^{\mathrm{a}}$ Lourdes & $E$ & 21 & & \\
\hline Llanos Ortiz de Landaluze, Armando & $E$ & 21 & & \\
\hline \multicolumn{5}{|c|}{ Autores no citados } \\
\hline Vaquero Lastres, Jacobo & $E$ & & & 5 \\
\hline Ramil Soneira, José Maria & $E$ & & & 4 \\
\hline Benito del Rey, Luis & $E$ & & & 3 \\
\hline Mancebo Dávalos, Julian & $E$ & & & 3 \\
\hline Rey Castiñeira, Xosefa & $E$ & & & 3 \\
\hline Sacristan de Lama, José David & $\mathrm{E}$ & & & 3 \\
\hline Santos, Manuel Farinha dos & $\mathrm{PT}$ & & & 3 \\
\hline Vázquez Rozas, Roberto & $E$ & & & 3 \\
\hline
\end{tabular}

Tabla 6. «Revistas y Series monográficas», «Congresos» y «Monografías»: (A) nacionalidad: AL alemana, E española, F francesa, I italiana y PT portuguesa; (B) ranking de autores por número de citas; (C) número de autocitas; (D) número de escritos.

caciones españolas, $63(47 \%)$ a las portuguesas y $27(20,2 \%)$ a las consideradas como ibéricas.

La nacionalidad de los coautores en los 44 do- cumentos españoles es: 105 españoles, 13 portugueses y 2 alemanes. Sólo hay un equipo hispano-luso (Querol et alii, 1975).

T. P., 54, n. ${ }^{\circ} 1,1997$ 
En los 63 documentos fuente portugueses, las nacionalidades se distribuyen así: 113 autores portugueses, 14 franceses, 10 españoles, 6 alemanes, 2 británicos y 2 de otros países.

En los 27 documentos restantes hay 35 autores españoles, 22 portugueses, 7 alemanes, 3 franceses, 1 británico y 2 de otros países. Sin embargo en la obra de Almagro Gorbea y Ruiz Zapatero (1992) la coautoría se da siempre entre autores de la misma nacionalidad. En este sentido las Actas del $1^{\circ}$ Congresso de Arqueologia Peninsular son el máximo exponente de la colaboración internacional en todas sus vertientes: sus 20 comunicaciones tienen firmantes de alguna de las nacionalidades siguientes: española, portuguesa, alemana, francesa, británica y otras.

\subsection{Lengua y nacionalidad}

Otros indicadores de la influencia de las diferentes tradiciones científicas son la nacionalidad de los firmantes y la lengua en la que publican, así como la lengua de la cita y la nacionalidad de su autor (Tab. 7).

\begin{tabular}{|c|c|c|c|c|c|c|c|c|c|c|c|c|c|}
\hline & \multicolumn{7}{|c|}{ Nacionalidad del primer firmante } & \multicolumn{6}{|c|}{ Lengua de los escritos } \\
\hline & PT & $E$ & $\mathrm{AL}$ & $F$ & I RU & OT & $?$ & PT & $E$ & $\mathrm{AL}$ & $\mathrm{F}$ & 1 & IN \\
\hline Arqueologia & 55 & 13 & 1 & 10 & 1 & 1 & 1 & 58 & 13 & & 8 & & 3 \\
\hline APort & 13 & 1 & 4 & 3 & & 1 & & 20 & & & 1 & & 1 \\
\hline Setúbal A. & 23 & 2 & & 2 & & & & 25 & 1 & & 1 & & \\
\hline TrabAntropEtnol & 19 & 10 & & 1 & & & & 19 & 10 & & 1 & & \\
\hline II CNA. Coimbra & 23 & 1 & 1 & 1 & & & & 24 & 1 & & 1 & & \\
\hline $1^{\circ}$ CAP. Porto & 21 & 13 & 2 & 1 & & 2 & & 22 & 15 & & & & 2 \\
\hline Alarcão (coord.) (1990) & 8 & & & & & & & 8 & & & & & \\
\hline TOTAL: 234 & 162 & 40 & 8 & 18 & 1 & 4 & 1 & 176 & 40 & & 12 & & 6 \\
\hline$B S A A$ & & 9 & & & & & & & 9 & & & & \\
\hline Gallaecia & & 20 & & 1 & & & & & 20 & & 1 & & \\
\hline Habis & & 12 & & & & & & & 12 & & & & \\
\hline$T P$ & & 9 & 1 & & & & & & 9 & & 1 & & \\
\hline Zephyrus & 2 & 31 & 2 & & & & & 2 & 33 & & & & \\
\hline XIII CNA. Huelva & 2 & 16 & & & & & & 2 & 16 & & & & \\
\hline XVII CNA . Logroño & 2 & 6 & & & & & & 2 & 6 & & & & \\
\hline XXII CNA. Vigo & 1 & 46 & 1 & & & & & 1 & 47 & & & & \\
\hline Jordá et alii (1986) & & 10 & & & & & & & 10 & & & & \\
\hline $\begin{array}{l}\text { Almagro Gorbea y Rulz } \\
\text { Zapatero (eds.) (1992) }\end{array}$ & 3 & 8 & & & & & & 3 & 8 & & & & \\
\hline TOTAL: 182 & 10 & 167 & 4 & 1 & & & & 10 & 170 & & 2 & & \\
\hline
\end{tabular}

Tabla 7. Documentos fuente: Nacionalidad del primer firmante: PT portuguesa, E española, AL alemana, $\mathrm{F}$ francesa, I italiana, RU británica, OT otras y ? no identificada. Lengua en la que están escritos: $\mathrm{PT}$ portuguesa, $\mathrm{E}$ española, $\mathrm{AL}$ alemana, F francesa, I italiana, IN inglesa, OT otras.
Como la coautoría es muy pequeña trabajamos con la nacionalidad del primer firmante del documento fuente.

Las fuentes portuguesas y españolas se diferencian significativamente en la presencia de firmantes extranjeros que, en las primeras, rondan la mitad del total, mientras en las segundas son el $8,2 \%$. En ambos casos la nacionalidad mejor representada es la del país vecino. En las portuguesas, además, hay firmantes de más de cuatro nacionalidades, destacando franceses y alemanes y en las españolas de dos.

Es llamativa la reducción de la presencia extranjera en la lengua de los escritos. Su absoluta correlación con la nacionalidad de los firmantes ibéricos contrasta con la ausencia del alemán y la reducción del francés. La presencia del inglés es mínima aunque es la lengua más manejada por autores de varias nacionalidades.

Todas las fuentes portuguesas, excepto la monografía, y siete españolas cuentan con colaboración extranjera.

Coherentemente con esos resultados, en las referencias bibliográficas, lengua y nacionalidad se corresponden de modo casi absoluto. Los escritos en inglés no distorsionan la muestra ya que, aunque hay autores no británicos que lo manejan, el volumen de citas de autores de otras nacionalidades que lo hacen es irrelevante estadísticamente.

La importancia de las citas extranjeras es distinta en los documentos fuente portugueses y españoles (Fig. 1.1). Sólo en los primeros, la cita extranjera supera a la del propio país.

Para averiguar si estos resultados representan una tendencia nacional o se deben al comportamiento diferencial de alguno de los tipos de documento hemos analizado las citas desde «Revistas y Series monográficas». Estos documentos facilitan los datos más amplios y variados sobre las fuentes de información manejadas por los arqueólogos.

Según estos resultados las fuentes de información de las revistas son las que definen la identidad entre la lengua de la cita y la nacionalidad del autor citado (Fig. 1.2). Los perfiles de las revistas ibéricas coinciden con los del conjunto de documentos fuente.

De la consideración de cada una de las revistas (Fig. 1.3) resulta la homogeneidad de las españolas a ese respecto y la influencia del elevado número de citas desde Arqueologia, en la conformación del perfil portugués. 

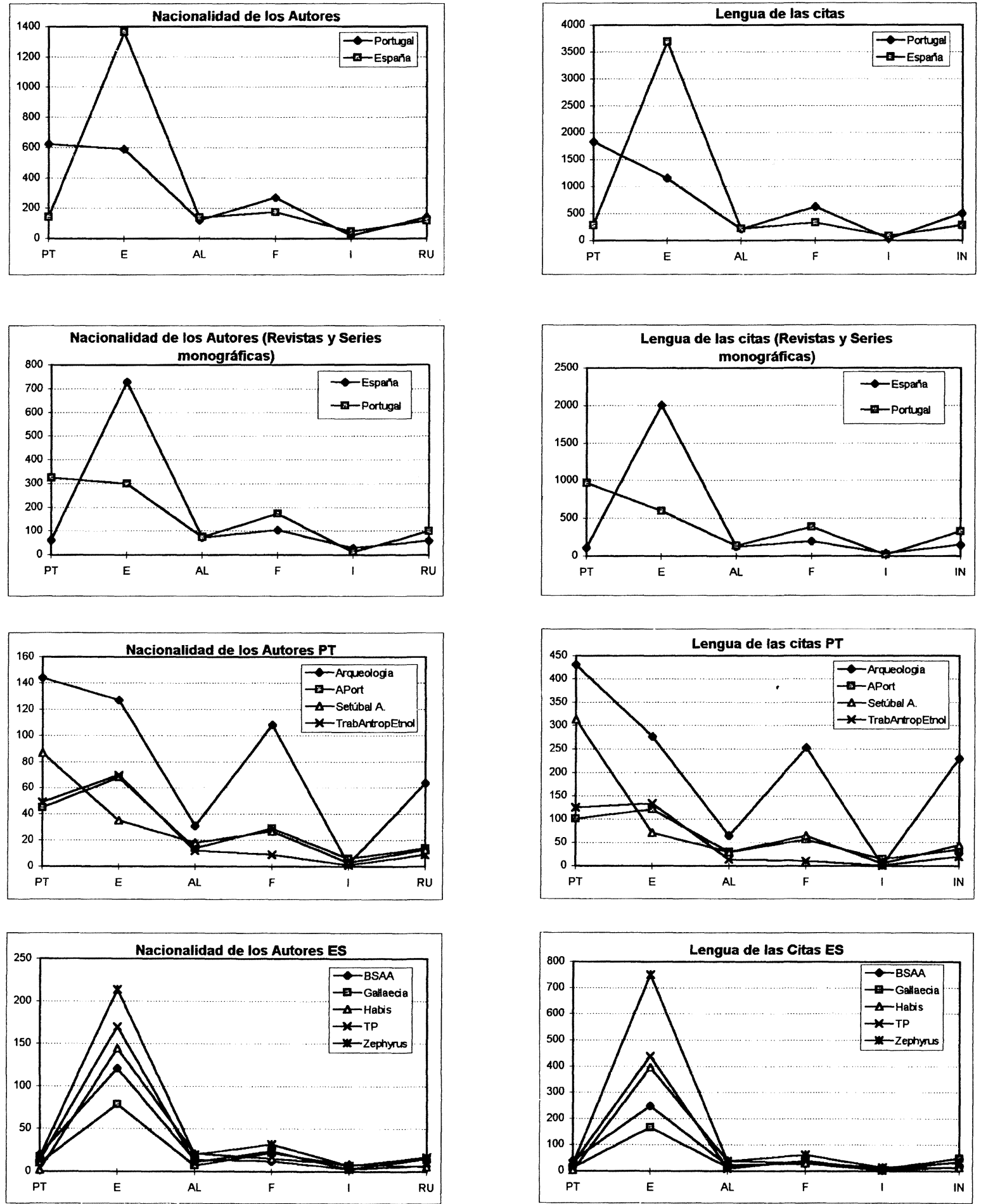

Fig. 1. Relación entre la lengua de la cita y la nacionalidad del autor citado en las publicaciones ibéricas: $\mathbf{1}$ totales desde todos los tipos de documentos; 2 totales desde las «Revistas y Series monográficas»; 3. totales desde cada una de las revistas portuguesas y españolas. Lengua: PT portuguesa, E española, AL alemana, $\mathrm{F}$ francesa, I italiana, IN inglesa. Nacionalidad: PT portuguesa, E española, AL alemana, F francesa, I italiana, RU británica.

T. P., 54, n. ${ }^{\circ} 1,1997$ 
La importancia relativa de las distintas nacionalidades y lenguas en las revistas es la siguiente: desde las españolas la mayoría de los autores citados son españoles que publican en español $(87,7 \%)$. A gran distancia figuran los artículos de autores franceses, ingleses, alemanes y portugueses cuyos valores e importancia relativa son análogos a los de las respectivas lenguas nacionales.

Desde las revistas portuguesas la cita a autores extranjeros y, correlativamente, a bibliografía en idioma no portugués es mayor. Aquí el español es la segunda lengua seguida, como en la revistas españolas, del francés, el inglés y el alemán.

Uno de los rasgos comunes a las revistas ibéricas es la importancia del francés y del alemán en relación con la influencia de la arqueología de estos países. Atribuimos el uso minoritario del italiano a la ausencia de la Arqueología Clásica de la muestra.

En conclusión, los arqueólogos portugueses evidencian una mayor apertura al exterior que sus colegas españoles tanto en el número de firmantes extranjeros en sus publicaciones, como en la cita extranjera. Esto tiene implicaciones metodológicas en la evaluación nacional e internacional del impacto de autores y publicaciones.

\subsection{Territorio}

El lugar de edición de las referencias bibliográficas precisa la importancia relativa de las tradiciones científicas desde cada uno de los documentos fuente.

Se ha estudiado a dos niveles a partir del país de publicación por tipo de documento (Tab. 8) y, en la Península Ibérica, a partir del lugar de publicación de las «Revistas y Series monográficas» (Tab. 9) y de la cartografía seleccionada. En este último caso se busca evaluar la regionalización de la investigación en cada país y redundar en la cuestión de la permeabilidad de la frontera.

Las Comunidades Autónomas agrupan los datos para todos los documentos fuente en un primer análisis. Además, dada la diferente extensión de las unidades administrativas ibéricas, el análisis se amplía a la Región en Portugal (Real, 1995: 22) y la provincia en España.

La tabla 2 presenta las citas a revistas españolas $(\mathrm{E})$, portuguesas $(\mathrm{F})$ y extranjeras $(\mathrm{H})$. Las diferencias respecto a los totales corresponden a los títulos vinculados a España y Portugal pero editados en otros países. El frecuente manejo de la revista alemana Madrider Mitteilungen se reconoce en la columna $\mathrm{G}$.

La suma de los totales de citas recibidas por las revistas editadas en cada región portuguesa y en cada provincia española es la fuente para confeccionar los mapas de las conexiones territoriales de las respectivas comunidades científicas. Aparecen las tres mas citadas o las cuatro cuando sus valores coinciden y siempre que se citan mas de una vez.

En cualquier caso, todas nuestras observaciones deben leerse recordando que la muestra incluye únicamente los artículos de Prehistoria y Protohistoria de la mitad occidental de la Península Ibérica.

\subsubsection{Distribución internacional de las citas}

El ámbito territorial de las publicaciones de la muestra incide en los datos obtenidos a partir del análisis de lengua y nacionalidad.

La cita a países por tipo de documento es bastante similar. La diferencia fundamental se encuentra en el número de países europeos citados con un máximo en las monografías y un mínimo en los congresos (Tab. 8). En cuanto a las fuentes ibéricas, coherentemente con los resultados del apartado 3.4, se citan muchas más publicaciones extranjeras desde las portuguesas.

Globalmente están representados todos los continentes, pero casi el $90 \%$ de las citas son a Europa (prácticamente todos los países). Como Portugal y España concentran la mayor parte de las referencias serán objeto de un estudio específico. Francia destaca claramente con el doble de citas que el Reino Unido y Alemania. Italia supera el centenar, mientras que el resto no pasa de la veintena.

En el caso de las fuentes ibéricas, las revistas se estudian en el apartado siguiente. Congresos y monografías son el $80 \%$ y el $70 \%$, respectivamente, del total de citas europeas a estos tipos de documentos.

\subsubsection{Regionalización de las citas}

La tabla 9 sugiere el sesgo que puede introducir el documento fuente -especialmente las monografías- en la cita a Regiones y Comunidades 


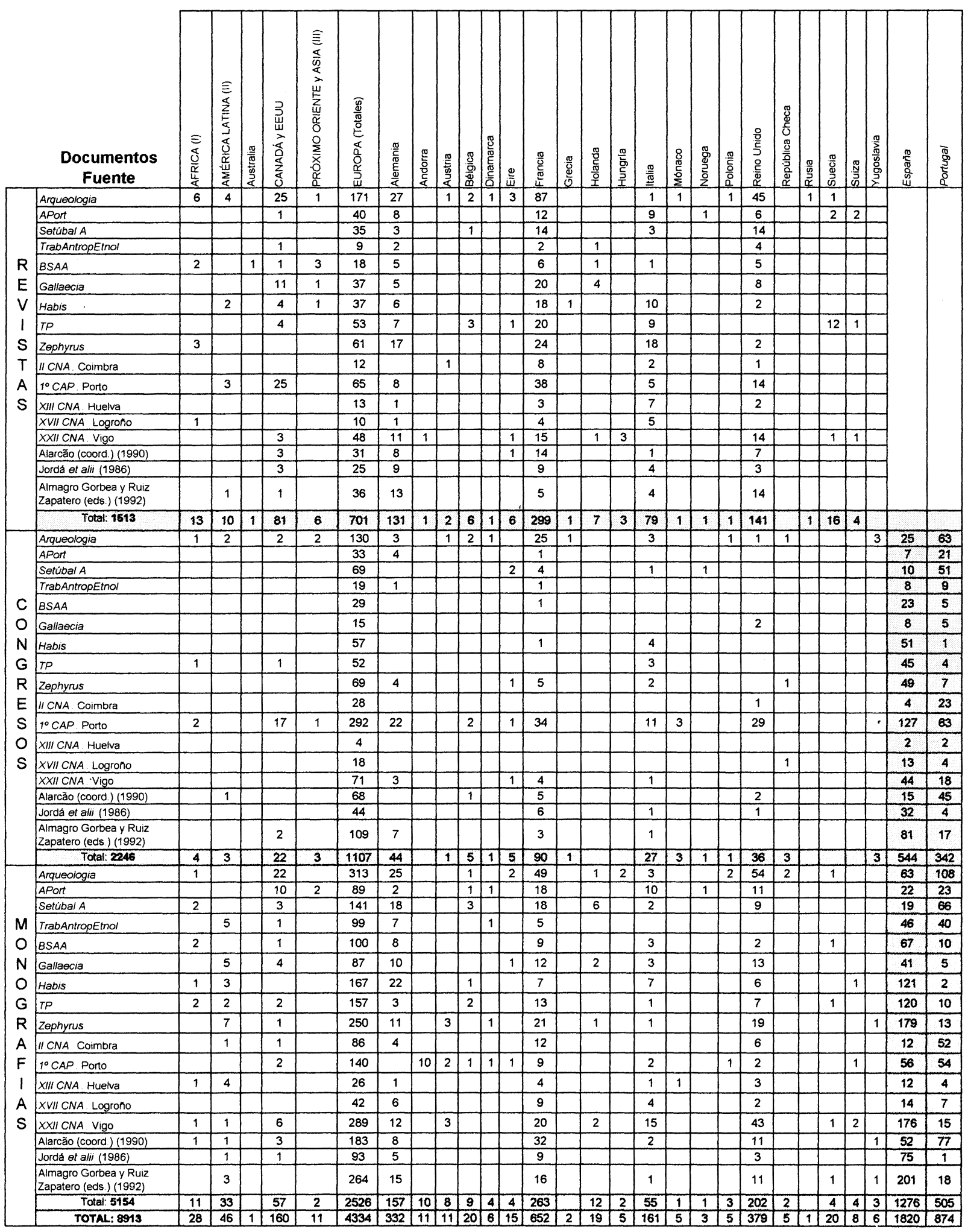

Tabla 8. Países de publicación por tipo de documento citado, salvo las revistas ibéricas. (I) Angola, Argelia, Egipto, Marruecos, República Sudafricana, Senegal, Sierra Leona y Túnez; (II) Argentina, Brasil, México y Venezuela; (III) India, Israel, Líbano y Turquía.

T. P., 54, n. ${ }^{\circ} 1,1997$ 


\begin{tabular}{|c|c|c|c|c|c|c|c|c|c|c|c|c|c|c|c|c|c|c|c|c|}
\hline Portugal & 离 & 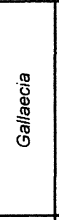 & 善 & $\stackrel{R}{F}$ & 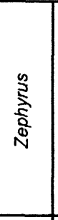 & 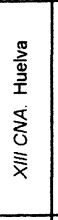 & 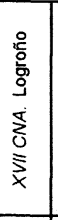 & $\begin{array}{l}8 \\
\stackrel{8}{5} \\
\dot{3} \\
0 \\
\overline{\bar{x}}\end{array}$ & 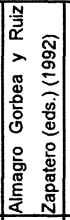 & 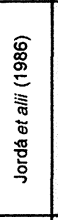 & 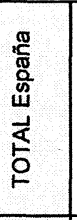 & 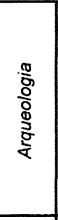 & $\begin{array}{l}\frac{5}{0} \\
\frac{0}{8}\end{array}$ & 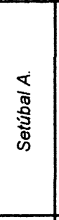 & 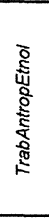 & 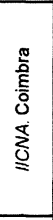 & $\begin{array}{l}\text { 옹 } \\
0 \\
0 \\
0 \\
\frac{0}{8} \\
0 \\
0\end{array}$ & 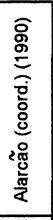 & 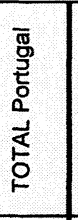 & 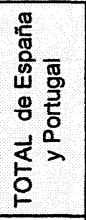 \\
\hline Alentejo & 1 & 1 & & 1 & 1 & 3 & & 1 & 2 & 2 & 12 & 1 & 5 & 46 & 2 & 2 & 29 & 11 & 96 & 108 \\
\hline Algarve & & & & & & & & & & & & & & & & & 2 & 2 & 4 & 4 \\
\hline Centro & 1 & 1 & & 1 & 1 & & 1 & 3 & 4 & & 12 & 26 & 4 & 6 & 1 & 8 & 25 & 4 & 74 & 86 \\
\hline Lisboa & 8 & 1 & 2 & 5 & 5 & 4 & 5 & 1 & 20 & 1 & 52 & 92 & 36 & 104 & 32 & 69 & 86 & 84 & 503 & 555 \\
\hline Norte & 17 & 11 & & 24 & 9 & 1 & 4 & 47 & 43 & 1 & 157 & 170 & 15 & 54 & 50 & 40 & 103 & 84 & 516 & 673 \\
\hline TOTAL & 27 & 14 & 2 & 31 & 16 & 8 & 10 & 52 & 69 & 4 & 233 & 289 & 60 & 210 & 85 & 119 & 245 & 185 & 1193 & 1426 \\
\hline \multicolumn{21}{|l|}{ España } \\
\hline Andalucía & 3 & & 41 & 3 & 17 & & 3 & 9 & 44 & 11 & 131 & 5 & & 2 & & & 12 & 2 & 21 & 152 \\
\hline Aragón & 2 & 2 & 1 & 5 & 8 & 3 & 3 & 7 & 9 & 9 & 49 & 3 & 1 & 1 & & & 10 & & 15 & 64 \\
\hline Asturias & & 1 & & 7 & 1 & 1 & & 6 & 3 & 4 & 23 & 1 & 1 & & & & 1 & & 3 & 26 \\
\hline Baleares & & & & 9 & & & & & & & 9 & & & & & & & & & 9 \\
\hline Canarias & & 1 & & 1 & & & & & & 2 & 4 & & & & & & & & & 4 \\
\hline Cantabria & 1 & & & 1 & 1 & & 1 & & 1 & 2 & 7 & 1 & & & & & 3 & & 4 & 11 \\
\hline Castilla-León & 41 & 8 & 13 & 20 & 164 & 8 & 13 & 23 & 47 & 31 & 368 & 35 & 14 & 8 & 1 & 2 & 28 & 9 & 97 & 465 \\
\hline Castilla-La Mancha & & & & 8 & 22 & & & 1 & & 1 & 32 & & & & & & 3 & & 3 & 35 \\
\hline Cataluña & 8 & 1 & 24 & 10 & 26 & & 1 & 7 & 20 & 16 & 113 & 3 & 5 & & 1 & 1 & 23 & 3 & 36 & 149 \\
\hline Comunidad Valenciana & 8 & & 1 & & 18 & & & & 6 & 16 & 49 & 5 & 6 & 1 & & & 3 & & 15 & 64 \\
\hline Extremadura & 4 & 1 & & 4 & 2 & & 1 & & 18 & & 30 & 1 & 1 & & & & 26 & & 28 & 58 \\
\hline Galicia & 8 & 67 & & 42 & 13 & 2 & 4 & 110 & 54 & 2 & 302 & 48 & 2 & & 42 & 5 & 22 & 9 & 128 & 430 \\
\hline La Rioja & & & & & & & & & 1 & & 1 & & & & & & & & & 1 \\
\hline Madrid & 72 & 16 & 123 & 121 & 199 & 26 & 38 & 66 & 138 & 56 & 855 & $61^{\circ}$ & 56 & 27 & 16 & 10 & 70 & 24 & 264 & 1119 \\
\hline Navarra & 1 & 1 & & & 8 & & & 1 & & 3 & 14 & & & & & & 1 & & 1 & 15 \\
\hline Pais Vasco & 4 & 5 & & 3 & 3 & 1 & & 7 & 11 & 8 & 42 & 8 & 1 & & 2 & & 13 & 1 & 25 & 67 \\
\hline Región de Murcia & & & & & & & 1 & & 1 & & 2 & & & & & & 1 & & 1 & 3 \\
\hline TOTAL & 179 & 117 & 205 & 265 & 498 & 49 & 75 & 289 & 422 & 165 & 2497 & 171 & 87 & 39 & 62 & 18 & 216 & 48 & 641 & 3138 \\
\hline
\end{tabular}

Tabla 9. Regiones portuguesas y Comunidades Autónomas españolas donde se editan las revistas citadas desde toda las fuentes.

Autónomas. Por ello nuestro primer comentario se basará en los totales.

La importancia de las publicaciones madrileñas en el conjunto de citas es clara. Las de la Región Norte y Lisboa se citan prácticamente la mitad de veces, las de Castilla-León y Galicia en torno a una tercera parte y las de Andalucía y Cataluña casi diez veces menos.

La situación varía si se consideran por separado las fuentes portuguesas y españolas. Desde éstas, las publicaciones madrileñas siguen concentrando la mayor parte de las referencias, seguidas de las localizadas en Castilla-León y Galicia. Las revistas de la Región Norte y Andalucía se consultan dos veces menos. Todas las Comunidades Autónomas se citan, pero las insulares, Cantabria, La Rioja y Murcia no llegan a diez referencias. La Región del Algarve es la única ausente.

Desde las fuentes portuguesas las publicaciones de la Región Norte y de Lisboa son las más citadas y se consultan de modo similar. Las de Madrid tienen casi la mitad de referencias que las anteriores y las de Galicia en torno a una quinta parte que no alcanzan las de Castilla-León, Alentejo y Centro. Las publicaciones de las Comunidades Autónomas insulares y La Rioja no se citan en absoluto y las de la Región del Algarve y las Comunidades Autónomas de Asturias, Cantabria, Castilla-La Mancha, Navarra y Murcia no pasan de 4 veces.

Los resultados indican el interés compartido de los investigadores de la mitad occidental de la Península Ibérica por las publicaciones editadas en Castilla-León, Galicia, Lisboa, Madrid y la Región Norte. La cita a revistas andaluzas y catalanas sólo desde las fuentes españolas se debería a la relevancia que la investigación española sobre Prehistoria y Protohistoria concede al mediodía peninsular y al carácter general de las revistas catalanas mas citadas (Rodríguez Alcalde et alii, 1996: 51) (Anexo). Las referencias a revistas de las Regiones del Alentejo y Centro sólo desde las portuguesas tendría que ver con el lugar de edición de la fuente o con su proximidad a él.

El análisis por Regiones y provincias, reitera la concentración de las citas (Fig. 2). Lisboa y Madrid, sedes de instituciones editoras de revis- 
tas de carácter general y de gran tradición, aparecen sistemáticamente (Rodríguez et alii, 1996: 48). El efecto centralizador de la capital no es la única razón que explica la importancia de Lisboa. La investigación regional también juega un papel en ese caso.

Otras unidades administrativas no están presentes, en gran parte, por no contar con publicaciones en la muestra ya que las que sí las tienen siempre suman un elevado número de referencias. Su orden respectivo varía desde cada documento fuente. En cualquier caso, el segundo lugar por número de citas $-\mathrm{y}$ con valores claramente distanciados de los del primero- corresponde a las revistas editadas en la propia provincia o Región. Sólo en Habis y Zephyrus ocupa el tercero (García Marín et alii, 1997).

Los documentos portugueses distribuyen la cita entre muchas provincias y la reiteran. Por contra los españoles la concentran en las mismas Regiones que los portugueses, limitándose el resto, en su caso, a una sola referencia.

La regionalización es evidente en la figura 2. $B S A A$ y Zephyrus, con los lugares de edición más próximos, citan las mismas provincias y $\mathrm{Re}$ giones. Mientras, Gallaecia y Habis, con los más alejados, sólo comparten la cita a publicaciones madrileñas. En cuanto a las revistas portuguesas, la Región Norte y Lisboa alternan su importancia por número de citas, dependiendo de su sede editorial. Las revistas del Algarve no reciben citas desde las revistas ibéricas y sólo 4 desde otros tipos de documentos portugueses (Tab. 9). Setúbal A. se ocupa significativamente del Alentejo.

Los contactos transfronterizos revelan otro aspecto de la regionalización. Las revistas de Salamanca son manejadas con mucha frecuencia desde APort y Setúbal A., en tanto que las revistas de Oporto recurren mayoritariamente a las de Pontevedra y La Coruña. Arqueologia, además, supera a las anteriores en la cita a Salamanca, lo que subraya la apertura de su línea editorial. Desde las revistas españolas, las de la Región Norte son las más consultadas. En Habis puede decirse que no hay referencia a Portugal.

La cita a revistas de Barcelona y Valencia es susceptible de varias interpretaciones: el carácter general de las mismas, la temática de algunos de sus artículos, la presencia de autores portugueses, o la de equipos que investigan fuera de la unidad administrativa donde publican.
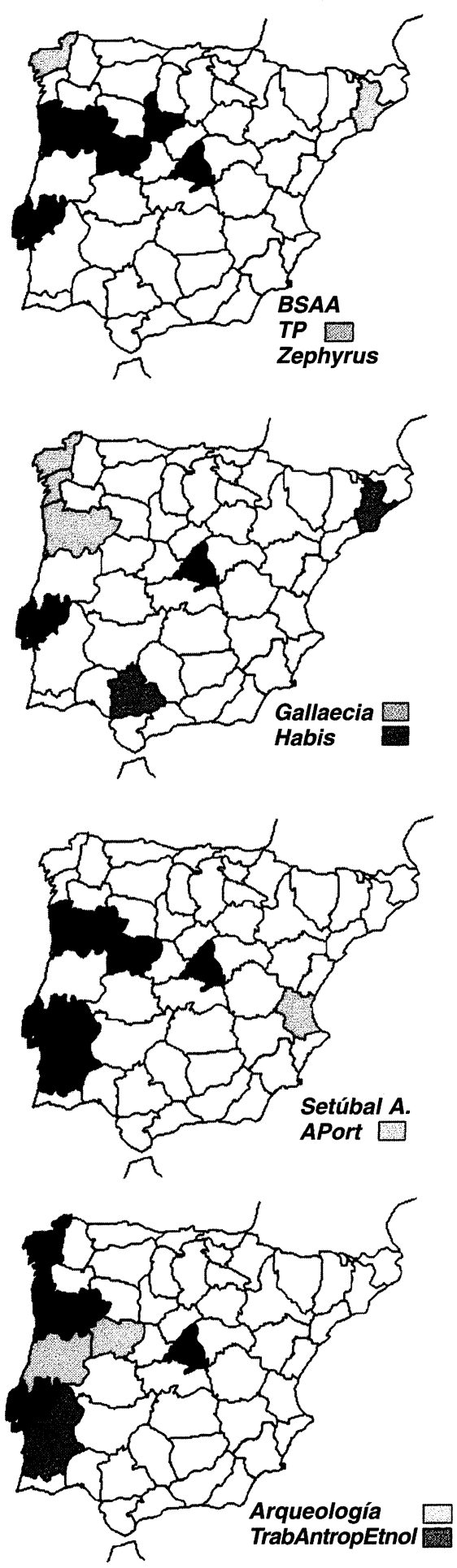

Fig. 2. Las Regiones portuguesas y las provincias españolas más citadas por los autores que publican en las revistas de la muestra. Aparecen en negro las compartidas. 


\subsubsection{Análisis de la cartografía}

La importancia que se da a la cartografía es mayor en las fuentes españolas que en las portuguesas a juzgar por la relación entre el número de mapas (Tab. 10) y el de escritos (Tab. 2:B). Por otra parte, las pautas de los autores a este respecto, es decir cuántos y cuáles incluyen, dependen del tipo de documento.

El análisis de la cartografía revela alguna de las formas de percepción del espacio por parte de los investigadores. La mayor parte de los mapas cuyo territorio no se puede identificar (categoría 0) (Tab. 10) se localiza en los congresos nacionales, sean estos portugueses o españoles. Los bajos valores de la misma en las monografías se deben bien a la práctica ausencia de mapas -en Portugal-, bien a la inclusión de toda la Península Ibérica (92\% de los mapas españoles).

En cuanto a las revistas, en un $44,5 \%$ de las españolas y un $71 \%$ de las portuguesas, la cartografía tampoco es identificable. Sin embargo, cuando lo es, los autores portugueses recurren más que los españoles a la inclusión del territorio peninsular.

El gran porcentaje de mapas inidentificables puede ser un reflejo del enorme localismo desde el que se concibe el objeto de estudio y la audiencia a la que va dirigida la investigación. Este hecho precisa la regionalización ya señalada.

Los pocos autores que introducen mapas identificables, en el caso de las Revistas fuente, si son portugueses siempre señalan la frontera. Ahora bien, la distribución del registro arqueoló-

\begin{tabular}{|c|c|c|c|c|c|c|c|c|c|c|c|}
\hline & \multicolumn{10}{|c|}{ Tipos de Mapas } & TOTAL \\
\hline Publicación & 01 & $\mathrm{~A} / \mathrm{B} 2 \mathrm{2A}$ & $2 \mathrm{~A}$ & & & 20 & $3 \mathrm{~A} 3 \mathrm{~B}$ & $3 C 30$ & 44 & & No de Mapas \\
\hline Revistas españolas & 37] & 72 & 5 & 4 & \begin{tabular}{l|l|l}
3 & 10 \\
\end{tabular} & & 81 & \begin{tabular}{|l|l|}
2 & 5 \\
\end{tabular} & 13 & & 83 \\
\hline Revistas portuguesas & 882 & $6 \longdiv { 1 5 1 9 }$ & 1 & & 15 & 3 & 10 & & 113 & 31 & 124 \\
\hline Congresos españoles & $26:$ & 33 & 4 & 1 & 12 & & 3 & & 2 & 4 & 43 \\
\hline Congresos portugueses & 42 & $2 \longdiv { 2 } 3$ & 1 & & & 1 & & & & 10 & 10 \\
\hline Congreso luso-español & 141 & 31211 & 11 & 1 & & & 4 & & 5 & 1 & 37 \\
\hline Monografila española & 4 & & 2 & & & & 41 - & & 45 & 1 & 50 \\
\hline Monografia portuguesa & 3 & 35 & & & & & 3 & & 1 & 1 & 10 \\
\hline Monografía luso-española & 7 & \begin{tabular}{l|l}
1 \\
\end{tabular} & & 1 & \begin{tabular}{|l|l|}
3 & 3 \\
\end{tabular} & & & 3 & & 1 & 13 \\
\hline
\end{tabular}

Tabla 10. Tipos de mapas contenidos en las publicaciones de la muestra. El mismo mapa puede aparecer en varias categorías. gico a uno y otro lado de la «raya» (categoría 3A) sugiere que ésta no se entiende como un límite para la investigación. El límite administrativo fundamental para los autores españoles es la provincia (categoría $2 \mathrm{C}$ ). En ese sentido no hay que ver con optimismo que, en los mapas de distribución, la «raya» no se dibuje (categoría 3A).

En cuanto a los resultados de este estudio en las monografías, el hecho de que sólo hayamos incluido una de cada país limita sus posibilidades de generalización. Es llamativo que Alarcão (coord.) (1990) incluya únicamente 10 mapas frente a los 50 de Jordá et alii (1986), de los cuales 41 presentan la dispersión de yacimientos en toda la Península Ibérica (categoría 3A).

\section{CONCLUSIONES}

El análisis bibliométrico de las publicaciones de Prehistoria y Arqueología de la muestra se revela como un poderoso instrumento de análisis de las distintas escuelas, lenguas y países, temas especialmente vulnerables a los sesgos nacionalistas. Los resultados obtenidos facilitan, además, indicadores sensibles a la especificidad de nuestro ámbito disciplinar.

La muestra es la clave de la calidad de los trabajos estadísticos. Una de las conclusiones de nuestro artículo es mostrar cómo sus parámetros son más relevantes que su tamaño: manejando 9.287 citas obtenemos resultados análogos a los de un estudio previo a partir de 33.474 (Rodríguez et alii, 1996: 39). Una muestra menor aumenta la calidad de la labor de documentación especializada indispensable para contrarrestar la heterogeneidad de los datos.

Los obstáculos son insalvables cuando proceden de la estructura de la propia tradición disciplinar. Así el incumplimiento de la periodicidad de las revistas limita la significación de los resultados de un estudio diacrónico y la escasa coautoría reduce la base de cualquier análisis del trabajo en equipo.

En el curso de la investigación hemos identificado algunas pautas de la comunicación científica que facilitan el análisis estadístico y otras que, por contra, introducen sesgos que es necesario controlar.

Los tipos de documentos determinan la información accesible. Las «Revistas y Series monográficas», por su propia estructura (periodicidad, 
pluralidad temática y de autores), proporcionan la más variada, significativa y con más posibilidades de comparación entre los datos fuente y las referencias. Por ejemplo, es el tipo más adecuado para indicar la influencia relativa de cada país. Las «Monografías» dispersan la cita y los «Congresos» facilitan muy poca información.

Un rasgo estructural de las revistas científicas es su especialización, que es inevitable sopesar para una correcta definición de la muestra y de los resultados de su análisis. Esto queda de manifiesto en la temática de «Revistas y Series monográficas» citadas.

Pensamos que puede generalizarse la importancia de la cita a las revistas publicadas en las capitales de los estados y que suelen corresponder con las de temática más general. Otro rasgo específico es la fuerte orientación local, advertida en estudios bibliométricos sobre las Ciencias Sociales y Humanidades (Nederhof y Zwaan, 1991: 332) y la Medicina (Navarro, 1996: 22), y que está muy marcada en Arqueología, tanto en España (Ruiz Zapatero, 1988; Rodríguez et alii, 1996: 49 y 55), como en Francia (Aubin y Jourdy, 1996). Sospechamos que dicha orientación es extensible a la Arqueología de otros países pero no conocemos bibliografía al respecto.

La concentración de la cita a autores y publicaciones simplifica la depuración de los datos ya que basta un tercio del total para tener garantizada una caracterización general. En este sentido, la escasa coautoría permite limitarse al primer firmante.

La práctica correspondencia entre lengua del escrito, país de publicación y nacionalidad del autor justifica manejar sólo esta última variable. Una observación complementaria es que, en los documentos fuente, los autores extranjeros aumentan la cita a la producción científica de su país de origen.

En definitiva, defendemos el estudio conjunto del documento fuente y sus referencias para la interpretación de los datos bibliométricos.

La vinculación de los autores a la edición del documento fuente y los límites cronológicos de la muestra introducen un primer sesgo en la evaluación del impacto de su producción. La primera favorece a los autores (compárense las columnas $\mathrm{P}$ y $\mathrm{Q}$ de la tabla 2). Los escritos más recientes están penalizados respecto a los más antiguos. Ello remite a las limitaciones ya advertidas sobre el Factor de Impacto de las revistas
(Rodríguez et alii, 1996: 54-55), extensibles al correspondiente a los autores. Así entre los autores más citados están los fundadores de la $\mathrm{Ar}$ queología peninsular que acumulan citas de todo el período. Además, en general, los autores más citados son los del propio país.

La lengua del escrito es otro condicionante de su impacto. Los autores que escriben en la lengua de un país experimentan una discriminación positiva desde los análisis bibliométricos nacionales y negativa desde los internacionales. Este segundo aspecto ha sido denunciado reiteradamente a propósito del sesgo que introduce la inclusión preferente de revistas escritas en lengua inglesa en el Science Citation Index y Social Science Citation Index (EEUU)(Navarro, 1996: 611; Gómez y Bordons, 1996: 23).

Nuestro trabajo redunda en el error que supone identificar la Ciencia con el impacto internacional de una determinada producción bibliográfica. Además, en el caso de la Arqueología ibérica, los datos hasta 1995 no justifican confundir «lo internacional» con la literatura en inglés.

Las pautas de comunicación que comparten los arqueólogos españoles y portugueses marcan fronteras y pasos entre las respectivas comunidades científicas.

La temática de las «Revistas y Series monográficas» ibéricas citadas muestra una gran especialización de la consulta bibliográfica: se manejan las de Prehistoria y Arqueología o misceláneas donde estas materias están siempre presentes. La aparición de títulos ajenos a las Humanidades y Ciencias Sociales se reduce a los de Geología y Ciencias Naturales. En general, la consulta de estas publicaciones se explicaría por la inclusión de artículos de Prehistoria antigua, sobre todo Paleolítico, o de las investigaciones de naturalistas y otros científicos aplicadas a la Arqueología.

La muestra presenta una baja coautoría ya constatada (Rodríguez et alii, 1996: 45-46). Las ventajas de la colaboración en la publicación son un tema tan discutido que, en los concursos para algunos departamentos del área de Prehistoria, se ha dividido la puntuación por artículo entre el número de autores o se ha rebajado (Universidades de Cantabria, Complutense...). En cualquier caso, la multidisciplinariedad, la especialización y el trabajo en equipo, de practicarse, no se reflejan en las publicaciones ibéricas de la muestra.

Las relaciones transfronterizas no caracterizan las publicaciones hispano-portuguesas. Sus ras- 
gos comunes más significativos procederían, más bien, de la tradición histórico cultural que configuró la Arqueología prehistórica y protohistórica ibérica (Martínez Navarrete, 1989; Lillios, 1995c). A ella se debe la orientación local de la investigación.

El territorio es la base para definir las culturas, los desplazamientos de sus miembros y la distribución de sus productos. El territorio satisface, además, las exigencias de objetividad y experiencia que se exigen al conocimiento científico. Su relevancia en la investigación queda reforzada asimismo al justificar el estudio del pasado por la recuperación y valoración del patrimonio histórico incluido en las fronteras nacionales.

La influencia alemana (Grünhagen, 1979; Luzón, 1995, Lillios, 1995c: 13-15) y, en menor medida, la francesa (Gran-Aymerich y GranAymerich, 1991) es responsable, en parte, de dicha tradición. Variables como el idioma de las citas, el lugar de edición de las revistas citadas (Rodríguez et alii, 1996: 42) y la presencia de autores de esas nacionalidades en las fuentes y en las citas la identifican bibliométricamente.

La cita recurrente a G. y V. Leisner en relación con el megalitismo en la muestra concuerda con el papel que se concede a este tema y en concreto a estos autores en la historiografía (Lillios, 1995a; Fabião, 1996: 95-96; Luzón, 1995:10-11). La implantación en España y Portugal del Instituto Arqueológico Alemán se manifiesta en la cita a sus publicaciones (Tab. 2:G) e investigadores y en la presencia de autores alemanes en las fuentes. Aunque publican en las lenguas ibéricas, sus referencias explican la presencia del alemán, idioma apenas manejado por autores de otras nacionalidades.

La influencia francesa se hace patente en que el país, como lugar de publicación, aparece siempre tras España y Portugal y en que su lengua es la más citada de las extrapeninsulares. Su inspiración destaca en la investigación de los períodos más antiguos de la Prehistoria. Su peso es mayor en las fuentes portuguesas. Sus indicadores son el número significativo de autores franceses, la existencia de publicaciones portuguesas en ese idioma y la importancia de la cita a publicaciones de Geología.

La baja intensidad de los contactos hispanoportugueses no excluye que los mejor representados, en ambos casos, sean los mantenidos con el país vecino. De hecho, ello es coherente con el localismo de la Arqueología. Casi todos los valores de los parámetros que hemos manejado para evaluar la comunicación entre los arqueólogos ibéricos están directamente relacionados con la proximidad entre fuente y cita. A pequeña escala, tenemos el gran porcentaje de mapas inidentificables. A una escala intermedia, la intensidad de las relaciones entre las regiones más próximas queda patente en publicaciones y autores citados. A mayor escala, las publicaciones del continente europeo concentran la cita y, por países, los más cercanos, geográficamente y/o por tradición científica.

Estos pasos transfronterizos no llegan a ocultar la «raya» fundamental entre los arqueólogos portugueses y españoles: unas pautas de comunicación científica mucho más abiertas al exterior por parte de los primeros. Los indicadores de esta apertura son la presencia extranjera en la cita, en los autores de los documentos fuente y en la lengua en que se escriben. Recordemos la llamativa ausencia de arqueólogos portugueses en el tercio de autores más citados en las publicaciones españolas.

Una investigación bibliométrica complementaria en fuentes, en primer término, francesas podría aclarar si esa pauta refleja el impacto de los arqueólogos portugueses o un sesgo nacionalista por parte de sus colegas españoles.

Otra manifestación de la personalidad de cada una de las comunidades de arqueólogos peninsulares es la especificidad de los ranking de citas a autores y publicaciones.

Insistimos, para finalizar, en que las diferencias advertidas nos parecen menos significativas, para entender la conceptualización de la Arqueología en España y Portugal, que la tradición científica común. Esta tradición, que favorece una forma actualista de entender el territorio, puede rastrearse en otros países dentro y fuera de Europa (Falkenhausen, 1995: 203; Kaiser, 1995: 108; Kohl y Tsetskhladze, 1995: 160-161).

No sabemos si otros factores que hemos detectado en la investigación arqueológica ibérica serían también generalizables. Pensamos en la «cortedad de miras» en el manejo de la bibliografía para la que sugerimos diferentes explicaciones. Con anterioridad a la década de los ochenta los estudios comparativos amplios eran una exigencia de cientificidad. Esta presión para manejar el registro arqueológico de otros países o zo- 
nas desaparece con la puesta en cuestión del paradigma difusionista. Paralelamente la descentralización administrativa ha promovido una introversión de la investigación en España. Quizá esta nueva situación política -que no se da en Portugal- influya en este comportamiento.

La investigación prehistórica ha ido ajustándose cada vez más a los límites administrativos actuales hasta el punto que su coincidencia con los de las culturas arqueológicas no se pone en cuestión. Ello puede ser una respuesta práctica a las ventajas económicas, administrativas y/o políticas que ello conlleva (Falkenhausen, 1995: 210-213 y 215). Pero en todo caso, los arqueólogos, al hacerlo, incurrimos en una grave falla metodológica que es nuestra responsabilidad específica denunciar.

De cualquier modo, la definición del ámbito de estudio no justifica la cita mayoritaria y reiterada a los autores y publicaciones mas cercanos. En este sentido nuestro artículo querría sumarse a las denuncias recientes sobre los sesgos de manipulación nacionalista de la práctica arqueológica implícitos en esa forma de conceptualizar y practicar el estudio de las sociedades del pasado.

\section{AGRADECIMIENTOS}

A Antonio Gilman Guillén por su traducción. A los colegas de las Universidades de Alcalá, Valladolid y del Instituto Arqueológico Alemán que nos dieron su opinión de expertos para la selección de la muestra. Al personal de la Biblioteca del Museo Arqueológico Nacional-«Colección de Prehistoria» del CSIC (Ministerio de Educación y Cultura) por su ayuda en la consulta de los fondos.

\section{BIBLIOGRAFÍA}

AlarCão, Jorge de (coord.) (1990): Nova História de Portugal. Vol. I. Portugal das Origens à Romanizaçao. Ed. Presença. Lisboa.

Almagro Gorbea, Martín y Ruiz Zapatero, Gonzalo (eds.)(1992): Paleoetnología de la Península Ibérica. Complutum Extra, 2-3. Madrid.

Aubin, Gerard y Jourdy, Dominique (1996): «Analyses et propositions à propos de la publication archéologique en France. Rapport sur les revues interrégionales d'Archéologie». Les Nouvelles de l'archéologie, 64: 5-17.

T. P., 54, n. ${ }^{\circ} 1,1997$
Chapman, Robert W. (1996): «Western Mediterranean Prehistory viewed from BARs». En David Davidson y Martin Henig (eds.): British Archaeological Reports. Past, present and future. Proceedings of a Conference held in Oxford in June 1994 to mark the twentieth anniversary of BAR. BAR. Oxford: 32-36.

FABIÃO, Carlos (1996): «Archaeology and nationalism: the portuguese case». En Margarita Díaz Andreu y Timothy Champion (eds.): Nationalism and Archaeology in Europe. UCL Press. London: 90-107.

FALKENHAUSEN, Lothar von (1995): «The regionalist paradigm in Chinese archaeology». En Philip L. Kohl y Clare Fawcett (eds.): Nationalism, politics, and the practice of Archaeology. Cambridge University Press. Cambridge: 198-217.

Ferreiro AlÁez, L. (1993): Bibliometría (análisis bivariante) Ed. EYPASA. Madrid.

García Marín, Ángeles; Sales Heredia, Patricia y RoMÁN RoMÁN, Adelaida (1996): «Evaluación de publicaciones periódicas en el ámbito de las Ciencias Sociales y Humanas. Estudio de los indicadores de calidad: el juicio de los pares y la difusión internacional». En Sistemas de Información: Balance de 12 años de jornadas y perspectivas de futuro. V Jornadas españolas de documentación automatizada (Cáceres, 17 a 19 de octubre 1996). Actas 2. FESABID, ABADMEX, Universidad de Extremadura. Cáceres: 994-1018

García Marín, Ángeles; Rodríguez Alcalde, Ángel L.; San Millán Bujanda, M. Jesús; Vicente Bobadilla, Gregorio de y Martínez NAVARRETE, M. ${ }^{a}$ Isabel (1997): «La frontera hispano-portuguesa a través del análisis bibliométrico de publicaciones de Prehistoria y Protohistoria». Actas del II Congreso de Arqueología Peninsular (Zamora, 1996). Fundación Rei Afonso Henriques, Zamora.

Gómez CARIDAD, Isabel y Bordons GANGAS, María (1996): «Limitaciones en el uso de los indicadores bibliométricos para la evaluación científica». Política Científica, 46: 21-26. Madrid.

GrAN-AYMERICH, Evelyne et Jean (1991): «Les échanges franco-espagnols et la mise en place des institutions archéologiques (1830-1939)». En Javier Arce y Ricardo Olmos (coord.): Historiografía de la Arqueología y de la Historia Antigua en España (Siglos XVIII$X X)$. Ministerio de Cultura. Madrid: 117-124.

GRÜNHAGEN, Wilhelm (1979): «Zur Geschichte der Abteilung Madrid des Deutschen Archäologischen Instituts von 1929 bis 1979». Das Deutsche Archäologische. Institut Geschichte und Dokumente, 3: 117-170.

JordÁ, Francisco; Pellicer, Manuel; Acosta, Pilar y Almagro Gorbea, Martin (1986): Historia de España. Vol. 1. Prehistoria. Ed. Gredos. Madrid.

KAISER, Timothy (1995): «Archaeology and ideology in southeast Europe». En Philip L. Kohl y Clare Fawcett (eds.): Nationalism, politics, and the practice of 
Archaeology. Cambridge University Press. Cambridge: 99-119.

Kohl, Philip L. y TseTskhladze, Gocha R. (1995): «Nationalism, politics, and the practice of Archaeology in the Caucasus». En Philip L. Kohl y Clare Fawcett (eds.): Nationalism, politics, and the practice of Archaeology. Cambridge University Press. Cambridge: $149-174$.

Kunst, Michael (coord.)(1995): Origens, Estruturas $e$ Relações das Culturas Calcolíticas da Península Ibérica. Actas das I Jornadas Arqueológicas de Torres Vedras (3-5 Abril 1987). Trabalhos de Arqueologia, 7. Lisboa.

Lillios, Katina T. (1995a): «Nationalism and Copper Age research in Portugal during the Salazar regime (19321974)». En Philip L. Kohl y Clare Fawcett (eds.): Nationalism, politics, and the practice of Archaeology. Cambridge University Press. Cambridge: 57-69.

- (1995b): «Introduction». En K.T. Lillios (ed.): The Origins of Complex Societies in Late Prehistoric Iberia. International Monographs in Prehistory, Ann. Arbor Michigan: V-VII.

- (1995c): «The Historiography of Late Prehistoric Portugal». En K.T. Lillios (ed.): The Origins of Complex Societies in Late Prehistoric Iberia. International Monographs in Prehistory, Ann. Arbor Michigan: 7-19.

LuZón Nogué, José M.a (1995): «Arqueología alemana en España y Portugal - una visión retrospectiva». Madrider Mitteilungen, 36. 1-11.

Martínez Navarrete, M. ${ }^{a}$ Isabel (1989): Una revisión crítica de la Prehistoria española: la Edad del Bronce como paradigma. Siglo XXI. Madrid.

Matanzo Caballero, Rosa M. ${ }^{a}$ (1982): «La comunicación de la información geográfica a través de mapas temáticos que respeten las leyes de la percepción visual». Geographica, XXIV:15-29.
NAVARRo, Fernando A. (1996): «El idioma de la medicina a través de las referencias bibliográficas de los artículos originales publicados en Medicina Clínica durante 50 años (1945-1995)». Medicina Clínica, 107: 608-613. Barcelona.

NEDERHOF, A.J. y ZwAAN, R.A. (1991): «Quality judgments of journals as indicators of research performance in the Humanities and the Social and Behavioral Sciences». Journal of the American Society for Information Science, 42(5): 332-340.

Oliveira, Eduardo Pires de (1985): Bibliografía Arqueológica Portuguesa (1970-1979). Instituto Português do Património Cultural, Departamento de Arqueologia. Lisboa.

Querol Fernández, M. ángeles; Lemos, Francisco de Sande; Monteiro, Jorge Pinho y Gomes, Mario Varela (1973): «El complejo de arte rupestre del Tajo (Portugal)». Actas del XIII Congreso Nacional de Arqueología (Huelva, 1973): 237-244. Zaragoza.

ReAL, Fernando C.S. (1995): «A Arqueologia portuguesa em mapas e números». $1^{\circ}$ Congresso de Arqueologia Peninsular (Porto, 1993). Actas VIII. Trabalhos de Antropologia e Etnologia, 35(4): 19-40. Porto.

Rodríguez Alcalde, Ángel L.; SÁnchez Nistal, José

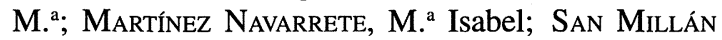
Bujanda, M. ${ }^{a}$ Jesús (1996): «Análisis bibliométrico de las revistas españolas de Prehistoria y Arqueología en los últimos diez años». Trabajos de Prehistoria, 53(1): 37-58. Madrid.

Ruiz ZaPATERo, Gonzalo (1988): «La prospección arqueológica en España: Pasado, presente y futuro». Arqueología Espacial, 12: 33-48.

- (1994): «¿Por una 'Europa arqueológica'unida?. Journal of European Archaeology». Trabajos de Prehistoria, 51(2): 191-193. 
Anexo. "RANKING" TOTAL DE REVISTAS Y SERIES MONOGRÁFICAS. Publicaciones con más de 10 citas recibidas.

ESPAÑA

156 Trabajos de Prehistoria. Madrid.

127 Zephyrus. Salamanca.

106 Archivo Español de Arqueología. Madrid.

105 Boletín del Seminario de Estudios de Arte y Arqueología. Valladolid.

100 Excavaciones Arqueológicas en España. Madrid.

99 Noticiario Arqueológico Hispánico. Madrid.

80 Madrider Mitteilungen. Mainz.

66 Bibliotheca Prehistorica Hispana. Madrid.

62 Memorias de la Junta Superior de Excavaciones y Antigüedades. Madrid.

51 Empuries. Barcelona.

49 Cuadernos de Estudios Gallegos. Santiago de Compostela.

43 Trabalhos de Antropologia e Etnologia. Porto.

41 Museo de Pontevedra. Pontevedra.

37 Revista de Guimarães. Guimarães.

35 Arqueologia. Porto.

34 Gallaecia. Santiago de Compostela.

32 Cuadernos de Prehistoria de la Universidad de Granada. Granada.

31 O Arqueologo Português. Lisboa.

30 Estudios de Arqueología Alavesa. Vitoria.

29 Arqueoloxía/Investigación. La Coruña.

27 Revista de Arqueología. Madrid.

24 Wad-Al-Hayara. Guadalajara.

23 Habis. Sevilla.

3 Pyrenae. Barcelona.

1 Acta Salmanticensia. Salamanca.

19 Boletín Auriense. Orense.

19 Boletín de la Asociación Española de Amigos de la Arqueología. Madrid.

18 Boletín de la Real Academia Gallega. La Coruña.

18 Huelva Arqueológica. Huelva.

18 Pontevedra Arqueológica. Pontevedra.

17 Acta Arqueológica Hispánica. Madrid.

16 Extremadura Arqueológica. Mérida-Cáceres.

16 Studia Archaeologica. Valladolid.

15 Brigantium. La Coruña.

5 Portugalia. Porto.

15 Saguntum. Papeles del Laboratorio de Arqueología de Valencia. Valencia.

14 Boletín de la Real Academia de la Historia. Madrid.

14 Munibe. San Sebastián.

13 Monografías del Museo Arqueológico de Valladolid. Valladolid.

13 Revista de Archivos, Bibliotecas y Museos. Madrid.

12 Caesaraugusta. Zaragoza.

12 Lancia. León.

12 Serie de Trabajos Varios. Servicio de Investigación Prehistórica. Valencia.

11 Actas y Memorias de la Sociedad Española de Antropología, Etnografía y Prehistoria. Madrid.
11 Anuario Arqueológico de Andalucía. Sevilla.

11 Archivo de Prehistoria Levantina. Valencia.

11 Arqueoloxía/Memorias. La Coruña.

11 Boletín de la Comisión Provincial de Monumentos Históricos y Artísticos de Lugo. Lugo.

11 Informes y Memorias de la Comisaría General de Excavaciones Arqueológicas. Madrid.

11 Revista de Estudios Extremeños. Badajoz.

10 Arqueología Espacial. Teruel.

10 Estudios. Seminario de Prehistoria, Arqueología e Historia Antigua. Zaragoza.

\section{PORTUGAL}

172 Arqueologia. Porto.

159 O Arqueologo Portugues. Lisboa.

93 Comunicações dos Serviços Geologicos de Portugal. Lisboa.

90 Revista de Guimarães. Guimarães.

79 Trabalhos de Antropologia e Etnologia. Porto.

72 Trabajos de Prehistoria. Madrid.

71 Setúbal Arqueologica. Setúbal.

65 Madrider Mitteilungen. Mainz.

55 Zephyrus. Salamanca.

48 Portugalia. Porto.

31 Arqueologia e História. Lisboa.

28 Pontevedra Arqueológica. Pontevedra.

27 Noticiario Arqueológico Hispánico. Madrid.

26 Bibliotheca Praehistorica Hispana. Madrid.

25 Excavaciones Arqueológicas en España. Madrid.

24 Archivo Español de Arqueología. Madrid.

24 Arquivo de Beja. Beja.

20 Conimbriga. Coimbra.

20 Cuadernos de Estudios Gallegos. Santiago de Compostela.

20 Memorias dos Serviços Geologicos de Portugal. Lisboa.

19 Boletín del Seminario de Estudios de Arte y Arqueologia. Valladolid.

19 Clio Revista do Centro de História da Universidade de Lisboa. Lisboa.

19 Informação Arqueologica. Lisboa.

18 Lucerna. Porto.

17 Museo de Pontevedra. Pontevedra.

17 Trabalhos de Arqueologia. Lisboa.

14 Ethnos. Lisboa.

12 Munibe. San Sebastián.

11 Anais da Académia Portuguesa da História. Lisboa.

11 Broteria Lisboa.

11 Empuries. Barcelona.

10 Extremadura Arqueológica. Mérida-Cáceres.

10 Gallaecia. Santiago de Compostela.

10 Memorias de la Comisión de Investigaciones Paleontológicas y Prehistóricas. Madrid. 